\title{
Spectrum and Energy Levels of Triply lonized Ytterbium
}

\author{
Jack Sugar and Victor Kaufman \\ Institute for Basic Standards, National Bureau of Standards, Washington, D.C. 20234 \\ Nissan Spector
}

Soreg Nuclear Research Center, Yavne 70600, Israel

January 19, 1978

\begin{abstract}
Spectra of $\mathrm{Yb}$ IV produced with a low voltage sliding spark discharge were photographed in the range of 700$2200 \AA$ with a $10.7 \mathrm{~m}$ normal incidence spectrograph. Wavelengths for 944 spectral lines identified as $\mathrm{Yb}$ IV were measured. Of these, 535 were classified as transitions between 95 even and 51 odd energy levels of the $4 f^{13}$, $4 f^{12} 5 d, 4 f^{12} 6 \mathrm{~s}$, and $4 f^{12} 6 p$ configurations. Values for the interaction parameters were derived by a least squares fit to the known levels.

Key words: Energy levels; parametric calculations; resonance lines; spectrum analysis; ytterbrium.
\end{abstract}

\section{Introduction}

The present work was carried out in connection with the interpretation of isoelectronic sequences related to various ionization states of tungsten and neighboring refractory elements. The sixth and seventh spectra of tungsten have been interpreted. [1] In the case of W VIII the $4 f^{14} 5 p^{5}$ and $4 f^{13} 5 p^{6}$ configurations compete for the ground state and it has not been established which configuration provides the lowest level. The highest analyzed member of this sequence is now $\mathrm{Yb}$ IV.

Interpreting the fourth spectra of the rare earths has become relatively straightforward. Transition arrays occur in compact groups at predictable wavelength regions. Accurate calculations of relative energy levels may be made, using highly predictable values for the interaction parameters. The challenge now lies in deducing enough levels to clarify the structure of these complicated configurations.

An initial analysis of $\mathrm{Yb}$ IV was given in which nineteen pairs of lines having the $4 f^{13}{ }^{2} F$ ground term interval were identified. [2] The upper levels were designated only as belonging to the $4 f^{12} 5 d$ configuration. The present analysis now provides 77 levels of $4 f^{12} 5 d, 18$ of $4 f^{12} 6 s$, and 49 of $4 f^{12} 6 p$. Nearly all levels built on the four lowest $4 f^{12}$ parent terms $\left({ }^{3} \mathrm{H},{ }^{3} \mathrm{~F},{ }^{1} \mathrm{G},{ }^{1} \mathrm{D}\right)$ were found. In addition, levels built on the ${ }^{1} I_{6}$ were determined but their connection with the rest of the system is based on only two lines because of the weakness of inter-parent transitions stemming from the high purity of this parent state. Stronger exposures are needed to confirm this connection.

The many known levels permitted us to make an empirical evaluation of all the Slater and spin-orbit single configuration radial intergrals as well as the two-body effective configuration interaction parameters. Explicit configuration interaction between $4 f^{12} 5 d$ and $4 f^{12} 6$ s was included in the calculation.

The results of this work were provided for inclusion in a compilation of rare earth energy levels [3] in advance of publication. We subsequently established 36 additional levels of the three excited configurations.

${ }^{1}$ Figures in brackets indicate literature references at the end of this paper.

\section{Experimental Procedures}

The light source for this work was the low voltage sliding spark originally described by one of us [4] as a modification of the Bockasten high voltage sliding spark. [5] It has been used to obtain the second through fifth spectra of rare earths by varying the peak spark current from 6-500 A, with a resulting sharp distinction among the spectra. For the measurements of $\mathrm{Yb}$ IV we used exposures made at $200 \mathrm{~A}$. The spark repetition rate was about $30 / \mathrm{s}$ with exposure times of two to five min. This produced relatively light exposures but clear groupings of transition arrays and easily measured plates.

All observations were made with the NBS $10.7 \mathrm{~m}$ normal incidence spectrograph in the range of observation of 700 2200 A. A few dozen lines at longer wavelength were reported by Bryant. [6] Some of these fit the known energy level structure but none provided new levels.

For reference wavelengths we used Cu II and Ge II obtained with a copper hollow cathode discharge operated at $0.5 \mathrm{~A}$ with a helium pressure of 2 torr.

\section{Wavelengths and Energy Levels}

Table I contains all the spectrum lines identified as Yb IV in the region we observed. The accuracy is estimated to be $\pm 0.005 \AA$. Wavelengths in air are given above $2000 \AA$. Intensities are visually estimated relative plate blackening. Line classifications are indicated by listing the two energy levels responsible for the transition, with subscripted $J$ values.

The energy levels of the $4 f^{12} 5 d$ configuration deduced earlier [2] from resonance lines were of little help initially in the new analysis. These transitions are governed by LS selection rules because of the 100 percent purity of the ${ }^{2} F$ ground state. For this reason they do not involve the lowest levels of $4 f^{12} 5 \mathrm{~d}$, which are predominantly of quartet character. Furthermore they have no correlation with $J_{1}$ j-coupling, the dominant coupling scheme of the $4 f^{12} 6 p$ configuration with which $4 f^{12} 5 d$ combines. 
The first regularities were found among the $4 f^{N} 6 s-4 f^{N} 6 p$ transitions (here $N=12$ ) because the coupling is clearly $J_{1} j$ in both cases, the lines are strong, relatively few, and confined to a small wavelength region. Also the energy intervals of these configurations may be calculated with great accuracy due to the small electrostatic effects and well defined spin-orbit parameters in the $4 f^{N}$ shell.

$W$ ith the discovery of the low structure of these configurations, we proceeded to find the low levels of $4 f^{12} 5 d$, and finally to tie them in with the levels from reference [2]. Predictions for missing levels were continually improved by fitting the calculations to the known levels.

The search for levels ended without producing those based primarily on the ${ }^{1} \mathrm{~S}$, or ${ }^{3} \mathrm{P}$ parent states of $4 f^{12}$. Because of the impurity of the eigenvectors of $4 f^{12} 5 d$ in $J_{1} j$-coupling, substantial components of the ${ }^{3} P$ parent occurs in several known levels. The ${ }^{1} I$ group were particularly intriguing because of their high $J$-values that lead to the classification of strong lines. An interval of $11.6 \mathrm{~cm}^{-1}$ was found among several pairs of strong unclassified lines. This matched almost exactly the predicted spacing between the pair of levels of $4 f^{12}\left({ }^{1} I_{6}\right) 6 s$. With this interpretation, the resulting $4 f^{12}\left({ }^{1} I_{6}\right) 6 p$ levels closely match their predicted values. However, the connection between these levels and the main system was elusive. Initially one level of the $4 f^{12}\left({ }^{1} I\right)$ group of $4 f^{12} 5 d$, the level at $120633.01 \mathrm{~cm}^{-1}$ with $J=9 / 2$, was found because it mixes strongly with a low-lying $4 f^{12}$ 6s level at $121008.13 \mathrm{~cm}^{-1}$. This enabled us to calculate the position of the entire $4 f^{12}\left({ }^{1} I\right)$ parent system very accurately. This group of levels was then nearly completely established, but its connection with the rest of the levels is based only on two observed transitions. These connect the $4 f^{12} 6 p$ level $\left({ }^{1} I_{6}, 1 / 2\right)_{13 / 2}$ with the $4 f^{12} 5 d$ levels $\left({ }^{3} H_{6}, 5 / 2\right)_{15 / 2}$ and $\left({ }^{3} H_{5}, 5 / 2\right)_{15 / 2}$. Though this is rather weak evidence our confidence in it is based on the discovery of similar lines of the isoelectronic ion, $\mathrm{Lu} \mathrm{V}$.

Table II contains all the experimentally deduced levels of $\mathrm{Yb}$ IV. The uncertainty in their values of $\pm 0.1 \mathrm{~cm}^{-1}$ is derived from a least squares adjustment to the observed lines. A few levels are given to one decimal place. These are determined only by short wavelength lines and have an uncertainty of $\pm 0.5 \mathrm{~cm}^{-1}$. A column is included that gives the number of lines classified by each level.

The levels are designated, with the exception of the ${ }^{2} F$ of $4 f^{13}$, in $J_{1} j$-coupling, which is the scheme of highest purity. For the $4 f^{12} 5 d$ configuration, $J_{1} j$-coupling is only slightly better than LS and many levels are highly mixed in both schemes.

Some levels of $4 f^{12} 6 s$ gave rise to resonance lines. This of course is due to mixing with the $4 f^{12} 5 d$ configuration. In a few cases the foreign configuration shows up as the second largest component of the eigenvector.

\section{Calculations}

Energy matrices were calculated for the $4 f^{12} 5 d$ and $4 f^{12} 6 s$ configurations with interaction, and the $4 f^{12} 6 p$ configuration. The effective electrostatic interactions for equivalent and inequivalent electrons were included. A brief description of the matrix calculations is given in reference [7]. These matrices were diagonalized initially with interpolated values of the parameters, which then were adjusted by a least squares fitting to the experimental energy levels.
Table III contains the fitted values for the parameters. Of the four "effective" electrostatic parameters of $4 f^{12} 5 d$ for inequivalent electrons, $D^{1}, D^{3}, X^{2}$, and $X^{4}$, only $D^{3}$ did not achieve a significant fitted value. The same result was found for $4 f^{13} 5 d$ in Lu IV [7]. In the case of $4 f^{12} 6 p$ the two parameters $D^{1}$ and $X^{3}$ are allowed. Here only $D^{1}$ is significant, as was true for $4 f^{13} 6 p$ of Lu IV. The effective parameters $\alpha, \beta$, and $\gamma$ of the $4 f^{12}$ core entered the Hamiltonian in the expression $\alpha L(L+1)+\beta Q+\gamma 12 G\left(G_{2}\right)$ where $Q$ is the seniority operator and $G\left(G_{2}\right)$ is Casimer's operator for the group $G_{2}$. The fixed value of $-1000 \mathrm{~cm}^{-1}$ for $\beta$ is estimated from the work of Goldschmit [9] on Ce III.

Configuration interaction between $4 f^{12} 5 d$ and $4 f^{12} 6 s$ is very weak. Only for very close levels is there any mixing. Two parameters are allowed but only the exchange parameter $R^{3}(f d, s f)$ is significant in the least squares fit.

Table IV and V contain the results of the calculation. The calculated levels are compared with the observed and the leading components of the eigenvectors are given in percent (squared components of eigenvector). The purity of the $4 f^{12} 5 d$

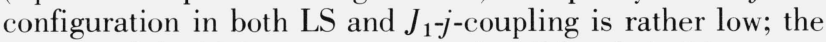
average of the highest percentages for all levels is 56 percent in $J_{1} j$-coupling and 50 percent in LS. The rms deviation for the $4 f^{12}(5 d+6 s)$ calculation is $58 \mathrm{~cm}^{-1}$ while for $4 f^{12} 6 p$ it is $20 \mathrm{~cm}^{-1}$.

\section{Conclusion}

Further progress will require the measurement of a stronger set of exposures. This should provide more connecting lines with the $4 f^{12}\left({ }^{1} I_{6}\right)$ nl system and allow for an extension of the analysis to include higher-lying configurations. The ionization energy is now determined by an interpolated value for the position of the $4 f^{12} 7 s$ configuration. [8] Some candidates were found for this and for $4 f^{12} 6 d$ but there was not sufficient certainty to report them here.

We wish to thank Dr. H. M. Crosswhite for his calculation of the $4 f^{12} 5 d$ configuration which led us to the $4 f^{12}\left({ }^{1} I_{6}\right)$ connection.

\section{References}

[1] Kaufman, V. and Sugar, J., Wavelengths, classifications, and ionization energies in the isoelectronic sequences from $\mathrm{Yb}$ II and $\mathrm{Yb}$ III through Bi XV and Bi XVI, J. Opt. Soc. Am. 66, 1019-1025 (1976).

[2] Kaufman, V. and Sugar, J., Resonance transition array of Yb IV, J. Opt. Soc. Am. 66, 439 (1976).

[3] Martin, W. C., Zalubas, R., and Hagan, L., Atomic Energy Levels-The Rare Earth Elements, Nat. Stand Ref. Data Ser., Nat. Bur. Stand. (U.S.), 60, in press.

[4] Sugar, J., Analysis of the third spectrum of praseodymium, J. Opt. Soc. Am. 53, 831-839 (1963).

[5] Bockasten, K., A study of C III by means of a sliding vacuum spark, Ark Fysik 9, 457-482 (1955).

[6] Bryant, B., The spectra of doubly and triply ionized ytterbium, Johns Hopkins Spectroscopic Report No. 21 (1961).

[7] Sugar J., and Kaufman, V., Fourth spectrum of lutetium, J. Opt. Soc. Am. 62, 562-570 (1972).

[8] Sugar J., and Reader, J., Ionization energies of double and triply ionized rare earths, J. Chem. Phys. 59, 2083-2089 (1973).

[9] Goldschmidt, Z. B., Thesis, Hebrew Univ. Jerusalem, Israel (1968). 
TABLE 1. Classified spectral lines of Yb IV.

Wavelengths longer than $2000 \AA$ are values in air.

\begin{tabular}{|c|c|c|c|c|c|c|c|c|}
\hline \multirow{2}{*}{$\begin{array}{c}\begin{array}{c}\text { Wavelength } \\
(\AA)\end{array} \\
2187.166\end{array}$} & \multirow{2}{*}{$\begin{array}{c}\text { Intensity } \\
150\end{array}$} & \multirow{2}{*}{$\begin{array}{c}\begin{array}{c}\text { Wavenumber } \\
\left(\mathrm{cm}^{-1}\right)\end{array} \\
45706.97\end{array}$} & Classification & \multirow{2}{*}{$\begin{array}{c}\begin{array}{c}\text { Wavelength } \\
(\AA)\end{array} \\
2036.870\end{array}$} & \multirow{2}{*}{$\begin{array}{c}\text { Intensity } \\
10\end{array}$} & \multirow{2}{*}{$\begin{array}{c}\begin{array}{c}\text { Wavenumber } \\
\left(\mathbf{c m}^{-1}\right)\end{array} \\
49079.16\end{array}$} & Classi & ification \\
\hline & & & $123710_{5 / 2}-169417_{7 / 2}^{\circ}$ & & & & & \\
\hline 2183.324 & 4000 & 45787.39 & $106801_{11 / 2}^{5 / 2}-152589_{11 / 2}^{\circ}$ & 2014.236 & 3 & 49630.58 & $112189_{9 / 2}$ & $-161820_{9 / 2}$ \\
\hline 2182.622 & 2 & 45802.11 & $130676_{9 / 2}-176478_{9 / 2}^{\circ}$ & 2004.472 & 8 & 49872.30 & $112189_{9 / 2}$ & $-16206 l_{11 / 2}^{\circ}$ \\
\hline 2180.558 & 2 & 45845.46 & $115974_{9 / 2}-161820_{9 / 2}^{\circ}$ & 1948.027 & 3 & 51333.99 & & \\
\hline 2177.528 & 3000 & 45909.25 & $115910_{11 / 2}-161820_{9 / 2}^{\circ}$ & 1945.254 & 80 & 51407.16 & & \\
\hline 2175.365 & 80 & 45954.89 & $130524_{7 / 2}-176478_{9 / 2}^{\circ}$ & 1918.639 & 20 & 52120.27 & & \\
\hline 2174.316 & 2 & 45977.06 & & 1917.987 & 50 & 52137.99 & $10680 l_{11 / 2}$ & $-158939_{9 / 2}$ \\
\hline 2172.158 & 2000 & 46022.73 & $123710_{5 / 2}-169733_{3 / 2}^{\circ}$ & 1915.155 & 1 & 52215.09 & & \\
\hline 2169.780 & 5 & 46073.17 & & 1892.778 & 1 & 52832.39 & $123710_{5 / 2}$ & $-176542_{7 / 2}^{\circ}$ \\
\hline 2169.116 & 3000 & 46087.27 & $115974_{9 / 2}-162061_{11 / 2}^{\circ}$ & 1892.405 & 10 & 52842.81 & & \\
\hline 2167.448 & 8 & 46122.73 & & 1886.073 & 15 & 53020.21 & & \\
\hline 2166.119 & 1000 & 46151.03 & $115910_{11 / 2}-162061_{11 / 2}^{\circ}$ & 1876.558 & 3 & 53289.05 & $122476_{5 / 2}$ & $-175765_{5 / 2}$ \\
\hline 2165.548 & 2500 & 46163.19 & $112772_{7 / 2}-158935_{7 / 2}^{\circ}$ & 1872.438 & 10 & 53406.30 & & \\
\hline 2164.382 & 800 & 46188.06 & $121008_{9 / 2}-167196_{9 / 2}^{\circ}$ & 1872.260 & 10 & 53411.38 & & \\
\hline 2163.878 & 600 & 46198.82 & & 1857.656 & 40 & 53831.27 & $130524_{7 / 2}$ & $-184355_{5 / 2}^{\circ}$ \\
\hline 2155.198 & 2 & 46384.86 & & 1841.034 & 500 & 54317.30 & $145128_{11 / 2}$ & $-199446_{9 / 2}^{0}$ \\
\hline 2154.249 & 200 & 46405.29 & $121008_{9 / 2}-167413_{7 / 2}^{\circ}$ & 1840.736 & 10 & 54326.09 & $123710_{5 / 2}$ & $-178036_{7 / 2}$ \\
\hline 2154.183 & 15000 & 46406.71 & $106801_{11 / 2}-153208_{13 / 2}^{\circ}$ & 1837.438 & 200 & 54423.60 & $112772_{7 / 2}$ & $-167196_{9 / 2}^{\circ}$ \\
\hline 2153.453 & 400 & 46422.44 & $120773_{9 / 2}-167196_{9 / 2}^{\circ}$ & 1833.584 & 150 & 54537.99 & & \\
\hline 2150.206 & 5 & 46492.54 & $122787_{3 / 2}-169279_{5 / 2}^{\circ}$ & 1831.184 & 1500 & 54609.47 & $145117_{13 / 2}$ & $-199726_{15 / 2}^{\circ}$ \\
\hline 2148.928 & 150 & 46520.18 & & 1829.480 & 3 & 54660.34 & & \\
\hline 2148.516 & 3000 & 46529.11 & $120667_{7 / 2}-167196_{9 / 2}^{\circ}$ & 1828.395 & 5 & 54692.77 & $122650_{7 / 2}$ & $-177343_{9 / 2}^{\circ}$ \\
\hline 2148.096 & 4000 & 46538.20 & $145128_{11 / 2}-191666_{13 / 2}^{\circ}$ & 1827.021 & 10 & 54733.90 & $112679_{5 / 2}$ & $-167413_{7 / 2}^{\circ}$ \\
\hline 2147.562 & 1000 & 46549.77 & $145117_{13 / 2}-191666_{13 / 2}^{\circ}$ & 1825.713 & 10 & 54773.12 & & \\
\hline 2145.560 & 1000 & 46593.20 & $137762_{3 / 2}-184355_{5 / 2}^{\circ}$ & 1824.219 & 2000 & 54817.97 & $112772_{7 / 2}$ & $-1675900_{5 / 2}$ \\
\hline 2144.769 & 20000 & 46610.38 & $105978_{13 / 2}-152589_{11 / 2}^{\circ / 2}$ & 1824.130 & 10 & 54820.65 & & \\
\hline 2143.892 & 3000 & 46629.45 & $122650_{7 / 2}-169279_{5 / 2}^{\circ}$ & 1821.101 & 60 & 54911.83 & $\left\{112679_{5 / 2}\right.$ & $-1675900_{5 / 2}$ \\
\hline 2143.425 & 3000 & 46639.61 & $120773_{9 / 2}-167413_{7 / 2}^{\circ}$ & & & & $\left\{137762_{3 / 2}\right.$ & $-192674_{5 / 2}^{\circ}$ \\
\hline 2142.195 & 2000 & 46666.38 & $130676_{9 / 2}-177343_{9 / 2}^{\circ}$ & 1820.936 & 1 & 54916.81 & & \\
\hline 2141.570 & 40 & 46680.00 & $145128_{11 / 2}-191808_{11 / 2}^{\mathrm{o} / 2}$ & 1819.024 & 3000 & 54974.53 & $123710_{5 / 2}$ & $-178684_{5 / 2}^{\circ}$ \\
\hline 2141.040 & 4000 & 46691.55 & $145117_{13 / 2}-191808_{11 / 2}^{\circ}$ & 1818.140 & 50 & 55001.26 & $97587_{11 / 2}$ & $-152589_{11 / 2}^{\circ}$ \\
\hline 2140.421 & 200 & 46705.06 & $137762_{3 / 2}-184467_{3 / 2}^{\circ}$ & 1817.583 & 2500 & 55018.12 & $106801_{11 / 2}$ & $-161820_{9 / 2}^{\circ}$ \\
\hline 2139.987 & 8000 & 46714.53 & & 1817.225 & 10 & 55028.95 & & \\
\hline 2138.532 & 2000 & 46746.31 & $-167413_{7 / 2}^{\circ}$ & 1817.106 & 20 & 55032.56 & & \\
\hline & & & $-158935_{7 / 2}^{\circ}$ & 1816.972 & 2 & 55036.62 & & \\
\hline 2138.354 & 5000 & 46750.20 & $112189_{9 / 2}-158939_{9 / 2}^{\circ}$ & 1816.762 & 20 & 55042.98 & $113049_{11 / 2}$ & $-168092_{9 / 2}^{\circ}$ \\
\hline 2137.715 & 30 & 46764.17 & & 1816.071 & 4000 & 55063.92 & $115910_{11 / 2}$ & $-170974_{13 / 2}^{\mathrm{o} / 2}$ \\
\hline 2137.575 & 3000 & 46767.23 & $-169417_{7 / 2}^{\circ}$ & 1814.953 & 1000 & 55097.84 & $120667_{7 / 2}$ & $-175765_{5 / 2}^{\circ}$ \\
\hline 2135.923 & 1500 & 46803.40 & $122476_{5 / 2}-169279_{5 / 2}^{\circ}$ & 1813.997 & 1000 & 55126.88 & $121008_{9 / 2}$ & $-176135_{11 / 2}^{\circ / 2}$ \\
\hline 2135.280 & 100 & 46817.49 & & 1813.843 & 6000 & 55131.56 & $105978_{13 / 2}$ & $-161110_{15 / 2}^{\circ}$ \\
\hline 2135.207 & 5000 & 46819.09 & $-177343_{9 / 2}^{\circ}$ & 1813.369 & 2 & 55145.97 & $122650_{7 / 2}$ & $-177796_{7 / 2}^{\circ}$ \\
\hline 2133.626 & 1500 & 46853.78 & $137501_{5 / 2}-184355_{5 / 2}^{\circ}$ & 1812.499 & 80 & 55172.44 & $137501_{5 / 2}$ & $-192674_{5 / 2}^{\circ}$ \\
\hline 2130.520 & 2 & 46922.08 & & 1810.602 & 1500 & 55230.24 & $130676_{9 / 2}$ & $-185907_{11 / 2}^{\circ}$ \\
\hline 2129.651 & 2000 & 46941.22 & $-169417_{7 / 2}^{\circ}$ & 1810.263 & 20 & 55240.59 & & \\
\hline 2129.434 & 800 & 46946.01 & $122787_{3 / 2}-169733_{3 / 2}^{\circ}$ & 1809.626 & 2500 & 55260.03 & $106801_{11 / 2}$ & $-162061_{11 / 2}^{\circ}$ \\
\hline 2128.840 & 1500 & 46959.10 & $123710_{5 / 2}-170669_{5 / 2}^{o^{2}}$ & 1809.549 & 100 & 55262.38 & $\left\{106557_{11 / 2}\right.$ & $-161820_{9 / 2}^{\circ}$ \\
\hline 2128.537 & 1500 & 46965.79 & $137501_{5 / 2}-184467_{3 / 2}^{\circ}$ & & & & $\left\{122787_{3 / 2}\right.$ & $-178049_{5 / 2}^{\circ / 2}$ \\
\hline 2126.207 & 1500 & 47017.25 & $-162991_{9 / 2}^{0 / 2}$ & 1809.140 & 4 & 55274.88 & & \\
\hline 2125.723 & 6000 & 47027.96 & $115974_{9 / 2}-163002_{11 / 2}^{\circ}$ & 1808.469 & 25 & 55295.39 & $130676_{9 / 2}$ & $-185972_{7 / 2}^{\circ}$ \\
\hline 2123.324 & 8000 & 47081.08 & $115910_{11 / 2}-162991_{9 / 2}^{\circ}$ & 1807.917 & 15 & 55312.27 & $112812_{9 / 2}$ & $-168124_{7 / 2}^{\circ}$ \\
\hline 2122.844 & 2500 & 47091.73 & $115910_{11 / 2}-163002_{11 / 2}^{\circ}$ & 1807.665 & 20 & 55319.98 & $\left\{122476_{5 / 2}\right.$ & $-177796_{7 / 2}^{\circ}$ \\
\hline 2121.592 & 2000 & 47119.51 & $130676_{9 / 2}-177796_{7 / 2}^{\circ}$ & & & & $\left\{112772_{7 / 2}\right.$ & $-168092_{9 / 2}^{\circ}$ \\
\hline 2120.910 & 800 & 47134.66 & & 1806.843 & 800 & 55345.15 & $130524_{7 / 2}$ & $-185869_{5 / 2}^{\circ}$ \\
\hline 2116.647 & 9000 & 47229.58 & $105978_{13 / 2}-153208_{13 / 2}^{\circ}$ & 1806.618 & 1000 & 55352.04 & $112772_{7 / 2}$ & $-168124_{7 / 2}^{\circ}$ \\
\hline 2115.423 & 250 & 47256.91 & $122476_{5 / 2}-169733_{3 / 2}^{5}$ & 1806.557 & 1500 & 55353.91 & & \\
\hline 2114.733 & 200 & 47272.32 & $-177796_{7 / 2}^{\circ}$ & 1806.318 & 1000 & 55361.23 & $120773_{9 / 2}$ & $-176135_{11 / 2}^{\circ}$ \\
\hline 2114.562 & 2 & 47276.15 & & 1805.500 & 200 & 55386.31 & $122650_{7 / 2}$ & $-178036_{7 / 2}^{\circ}$ \\
\hline 2112.642 & 200 & 47319.11 & $-168092_{9 / 2}^{\circ}$ & 1805.067 & 20 & 55399.60 & $122650_{7 / 2}$ & $-178049_{5 / 2}^{\circ}$ \\
\hline 2111.231 & 250 & 47350.73 & $-168124_{7 / 2}^{\circ}$ & 1804.914 & 20 & 55404.30 & & \\
\hline 2111.144 & 1 & 47352.68 & & 1803.486 & 600 & 55448.17 & $130524_{7 / 2}$ & $-185972_{7 / 2}^{\circ}$ \\
\hline 2110.862 & 100 & 47359.00 & & 1802.752 & 80 & 55470.74 & $121008_{9 / 2}$ & $-176478_{9 / 2}^{\circ}$ \\
\hline 2110.829 & 100 & 47359.74 & $130676_{9 / 2}-178036_{7 / 2}$ & 1802.326 & 50 & 55483.85 & & \\
\hline 2110.610 & 1 & 47364.66 & & 1801.735 & 8 & 55502.05 & $120633_{9 / 2}$ & $-176135_{11 / 2}^{\circ}$ \\
\hline 2108.228 & 5 & 47418.17 & & 1801.668 & 2000 & 55504.12 & $106557_{11 / 2}$ & $-162061_{11 / 2}^{\circ}$ \\
\hline 2106.484 & 3000 & 47457.42 & $120667_{7 / 2}-168124_{7 / 2}^{\circ}$ & 1800.679 & 1 & 55534.60 & $121008_{9 / 2}$ & $-176542_{7 / 2}^{\circ}$ \\
\hline 2099.844 & 1 & 47607.47 & & 1800.205 & 10 & 55549.22 & & \\
\hline 2091.929 & 1 & 47787.57 & & 1799.844 & 100 & 55560.37 & $122476_{5 / 2}$ & $-178036_{7 / 2}^{\circ}$ \\
\hline 2088.984 & 2 & 47854.93 & & 1799.415 & 200 & 55573.61 & $122476_{5 / 2}$ & $-178049_{5 / 2}^{\circ}$ \\
\hline 2087.788 & 1000 & 47882.35 & $-170669_{5 / 2}^{\rho}$ & 1798.725 & 800 & 55594.93 & $123710_{5 / 2}$ & $-179305_{7 / 2}^{\circ}$ \\
\hline 2086.616 & 3 & 47909.24 & $-179305_{7 / 2}^{\circ}$ & 1798.645 & 20 & 55597.40 & & \\
\hline 2081.832 & 10 & 48019.32 & $122650_{7 / 2}-170669_{5 / 2}^{2}$ & 1795.169 & 80 & 55705.06 & $120773_{9 / 2}$ & $-176478_{9 / 2}^{\circ}$ \\
\hline 2063.010 & 4 & 48457.37 & & 1794.998 & 1 & 55710.36 & & \\
\hline 2056.424 & 2 & 48612.54 & $-169279_{5 / 2}$ & 1793.113 & 2 & 55768.93 & $120773_{9 / 2}$ & $-176542_{7 / 2}^{\circ}$ \\
\hline 2046.004 & 30 & 48860.08 & $122650_{7 / 2}-171510_{7 / 2}$ & 1792.582 & 600 & 55785.45 & $130676_{9 / 2}$ & $-186462_{9 / 2}^{\circ}$ \\
\hline
\end{tabular}


TABLE 1. Classified spectral lines of $\mathrm{Yb}$ IV.

Wavelengths longer than $2000 \AA$ are values in air-continued

\begin{tabular}{|c|c|c|c|c|c|c|c|c|c|}
\hline $\begin{array}{c}\text { Wavelength } \\
(\AA)\end{array}$ & Intensity & $\begin{array}{l}\text { Wavenumber } \\
\left(\mathrm{cm}^{-1}\right)\end{array}$ & Classific & cation & $\begin{array}{c}\text { Wavelength } \\
(\AA)\end{array}$ & Intensity & $\begin{array}{l}\text { Wavenumber } \\
\left(\mathrm{cm}^{-1}\right)\end{array}$ & Classif & fication \\
\hline 1791.736 & 400 & 55811.79 & $120667_{7 / 2}$ & $-176478_{9 / 2}^{\circ}$ & 1633.465 & 1 & 61219.55 & $106801_{11 / 2}$ & $-168021_{11 / 2}^{\circ}$ \\
\hline 1791.301 & 1 & 55825.34 & & & 1631.941 & 100 & 61276.72 & $9193 l_{13 / 2}$ & $-153208_{13 / 2}^{\circ}$ \\
\hline 1791.085 & 6000 & 55832.07 & $112189_{9 / 2}$ & $-168021_{11 / 2}^{\circ}$ & 1631.578 & 1 & 61290.35 & & \\
\hline 1791.045 & 2000 & 55833.32 & $10680 l_{11 / 2}$ & $-162635_{13 / 2}^{\circ}$ & 1631.458 & 20 & 61294.86 & & \\
\hline 1790.249 & 400 & 55858.15 & & & 1629.371 & 2 & 61373.37 & $106039_{7 / 2}$ & $-167413_{7 / 2}^{\circ}$ \\
\hline 1790.178 & 1500 & 55860.36 & & & 1629.107 & 4 & 61383.32 & & \\
\hline 1789.720 & 2000 & 55874.66 & $122650_{7 / 2}$ & $-178524_{9 / 2}^{\circ}$ & 1627.458 & 8 & 61445.51 & & \\
\hline 1789.700 & 1000 & 55875.28 & $120667_{7 / 2}$ & $-176542_{7 / 2}^{\circ}$ & 1624.851 & 20 & 61544.10 & $107873_{5 / 2}$ & $-169417_{7 / 2}^{\circ}$ \\
\hline 1788.993 & 40 & 55897.36 & $122787_{3 / 2}$ & $-178684_{5 / 2}^{\circ}$ & 1621.833 & 4 & 61658.62 & $117026_{5 / 2}$ & $-178684_{5 / 2}^{\circ}$ \\
\hline 1788.800 & 500 & 55903.39 & $112189_{9 / 2}$ & $-168092_{9 / 2}^{\circ}$ & 1620.006 & 100 & 61728.16 & $105685_{7 / 2}^{0 / 2}$ & $-167413_{7 / 2}^{\circ}$ \\
\hline 1788.740 & 150 & 55905.27 & & & 1619.303 & 200 & 61754.96 & $90834_{11 / 2}$ & $-152589_{11 / 2}^{\circ}$ \\
\hline 1788.644 & 600 & 55908.27 & $137501_{5 / 2}$ & $-193409_{7 / 2}$ & 1619.094 & 20 & 61762.93 & & \\
\hline 1788.130 & 250 & 55924.34 & & & 1617.795 & 250 & 61812.52 & & \\
\hline 1787.687 & 200 & 55938.20 & $130524_{7 / 2}$ & $-186462_{9 / 2}^{\circ}$ & 1615.967 & 2 & 61882.45 & & \\
\hline 1787.466 & 85 & 55945.12 & & & 1615.369 & 1 & 61905.36 & & \\
\hline 1785.476 & 200 & 56007.47 & & & 1615.349 & 1 & 61906.12 & $105685_{7 / 2}$ & $-167590_{5 / 2}^{\circ}$ \\
\hline 1784.616 & 50 & 56034.46 & $122650_{7 / 2}$ & $-178684_{5 / 2}^{\circ}$ & 1615.197 & 20 & 61911.95 & & \\
\hline 1784.568 & 80 & 56035.97 & & & 1614.917 & 6 & 61922.68 & & \\
\hline 1783.071 & 200 & 56083.01 & $105978_{13 / 2}$ & $-16206 \mathrm{l}_{11 / 2}^{\circ}$ & 1614.886 & 2 & 61923.87 & & \\
\hline 1782.880 & 200 & 56089.02 & $102850_{11 / 2}$ & $-158939_{9 / 2}^{\circ}$ & 1612.910 & 1 & 6.1999 .73 & & \\
\hline 1779.676 & 600 & 56190.00 & $10680 l_{11 / 2}$ & $-16299 \mathrm{I}_{9 / 2}^{\circ}$ & 1612.535 & 80 & 62014.15 & $131395_{7 / 2}$ & $-193409_{7 / 2}^{\circ}$ \\
\hline 1779.335 & 2000 & 56200.77 & $10680 l_{11 / 2}$ & $-163002_{11 / 2}^{\sigma}$ & 1612.119 & 6 & 62030.16 & & \\
\hline 1778.201 & 2000 & 56236.61 & $115974_{9 / 2}$ & $-17221 l_{11 / 2}^{\circ}$ & 1611.795 & 8 & 62042.62 & $105978_{13 / 2}$ & $-168021_{11 / 2}^{\circ}$ \\
\hline 1776.185 & 2000 & 56300.44 & $115910_{11 / 2}$ & $-17221 l_{11 / 2}^{\mathrm{o}}$ & 1610.946 & 4 & 62075.32 & & \\
\hline 1775.094 & 2 & 56335.04 & $121008_{9 / 2}$ & $-177343_{9 / 2}^{\circ}$ & 1610.772 & 60 & 62082.03 & & \\
\hline 1772.824 & 20 & 56407.17 & & & 1610.660 & 50 & 62086.34 & & \\
\hline 1767.740 & 2 & 56569.40 & $120773_{9 / 2}$ & $-177343_{\mathbf{9} / \mathbf{2}}^{\circ}$ & 1610.024 & 100 & 62110.87 & & \\
\hline 1765.381 & 60 & 56644.99 & $112772_{7 / 2}$ & $-169417_{7 / 2}^{0}$ & 1609.806 & 250 & 62119.28 & & \\
\hline 1765.026 & 4000 & 56656.38 & $105978_{13 / 2}$ & $-162635_{13 / 2}^{\circ}$ & 1608.647 & 1 & 62164.04 & & \\
\hline 1764.783 & 20 & 56664.19 & & & 1608.529 & 1 & 62168.60 & & \\
\hline 1764.412 & 3 & 56676.10 & $120667_{7 / 2}$ & $-177343_{\mathbf{9} / \mathbf{2}}^{\circ}$ & 1605.673 & 30 & 62279.18 & $117026_{5 / 2}$ & $-179305_{7 / 2}^{\circ}$ \\
\hline 1761.670 & 500 & 56764.32 & $145128_{11 / 2}$ & $-201893_{11 / 2}^{\circ}$ & 1605.439 & 1 & 62288.25 & & \\
\hline 1761.310 & 2 & 56775.92 & $145117_{13 / 2}$ & $-201189_{11 / 2}^{9}$ & 1604.163 & 20 & 62337.80 & & \\
\hline 1759.659 & 15 & 56829.19 & $122476_{5 / 2}$ & $-179305_{7 / 2}^{\circ}$ & 1603.682 & 2 & 62356.50 & & \\
\hline 1754.892 & 8 & 56983.56 & & & 1603.226 & 250 & 62374.23 & $90834_{11 / 2}$ & $-153208_{13 / 2}^{\circ}$ \\
\hline 1753.658 & 60 & 57023.66 & $105987_{13 / 2}$ & $-163002_{11 / 2}^{\circ}$ & 1602.919 & 30 & 62386.18 & & \\
\hline 1753.029 & 10 & 57044.12 & & & 1602.454 & 2 & 62404.28 & & \\
\hline 1750.141 & 50 & 57138.25 & & & 1601.556 & 10 & 62439.27 & $105685_{7 / 2}$ & $-168124_{7 / 2}^{\circ}$ \\
\hline 1749.161 & 40 & 57170.26 & $145128_{11 / 2}$ & $-202299_{13 / 2}^{\circ}$ & 1600.294 & 40 & 62488.51 & & \\
\hline 1748.807 & 800 & 57181.83 & $145117_{13 / 2}$ & $-202299_{13 / 2}^{\circ}$ & 1600.055 & 6 & 62497.85 & & \\
\hline 1748.366 & 40 & 57196.26 & & & 1600.022 & 15 & 62499.14 & & \\
\hline 1744.281 & 6 & 57330.21 & & & 1599.376 & 20 & 62524.38 & & \\
\hline 1744.029 & 1 & 57338.49 & & & 1598.967 & 100 & 62540.37 & $115984_{7 / 2}$ & $-178524_{9 / 2}^{\circ}$ \\
\hline 1740.702 & 50 & 57448.08 & & & 1598.562 & 20 & 62556.22 & & \\
\hline 1731.569 & 5 & 57751.09 & $120773_{9 / 2}$ & $-178524_{9 / 2}^{\circ}$ & 1598.390 & 150 & 62562.95 & & \\
\hline 1729.480 & 1 & 57820.84 & & & 1598.077 & 600 & 62575.20 & & \\
\hline 1727.200 & 300 & 57897.17 & $112772_{7 / 2}$ & $-170669_{5 / 2}$ & 1597.892 & 1 & 62582.45 & & \\
\hline 1723.616 & 15 & 58017.56 & $120667_{7 / 2}$ & $-178684_{5 / 2}^{\circ}$ & 1597.694 & 80 & 62590.20 & & \\
\hline 1721.681 & 1 & 58082.76 & & & 1597.455 & 50 & 62599.57 & & \\
\hline 1717.217 & 1 & 58233.75 & & & 1596.640 & 30 & 62631.52 & & \\
\hline 1709.021 & 2 & 58513.03 & & & 1595.819 & 30 & 62663.74 & & \\
\hline 1706.228 & 15 & 58608.81 & $110808_{7 / 2}$ & $-169417_{7 / 2}^{0}$ & 1595.024 & 40 & 62694.98 & & \\
\hline 1705.367 & 10 & 58638.40 & $120667_{7 / 2}$ & $-179305_{7 / 2}^{\circ}$ & 1594.963 & 1 & 62697.37 & & \\
\hline 1700.755 & 5 & 58797.41 & & & 1594.569 & 800 & 62712.87 & & \\
\hline 1695.592 & 10 & 58976.45 & $120328_{5 / 2}$ & $-179305_{7 / 2}^{\circ}$ & 1594.005 & 300 & 62735.06 & & \\
\hline 1685.294 & 10 & 59336.82 & & & 1592.721 & 80 & 62785.63 & & \\
\hline 1680.201 & 3 & 59516.68 & $117026_{5 / 2}$ & $-176542_{7 / 2}^{\circ}$ & 1592.670 & 10 & 62787.64 & & \\
\hline 1674.059 & 3 & 59735.05 & & & 1592.456 & 200 & 62796.08 & $107873_{5 / 2}$ & $-170669_{5 / 2}^{\circ}$ \\
\hline 1670.539 & 2 & 59860.91 & $110808_{7 / 2}$ & $-170669_{5 / 2}$ & 1592.074 & 400 & 62811.15 & & \\
\hline 1653.413 & 1 & 60480.95 & & & 1591.390 & 100 & 62838.14 & & \\
\hline 1651.310 & 2 & 60557.98 & $115984_{7 / 2}$ & $-176542_{7 / 2}^{\circ}$ & 1591.112 & 1 & 62849.12 & & \\
\hline 1645.542 & 100 & 60770.25 & $117026_{5 / 2}$ & $-177796_{7 / 2}^{\circ}$ & 1591.025 & 80 & 62852.56 & & \\
\hline 1644.426 & 1 & 60811.49 & $130855_{15 / 2}$ & $-191666_{13 / 2}^{\circ}$ & 1590.641 & 60 & 62867.73 & & \\
\hline 1643.071 & 1 & 60861.64 & & & 1590.501 & 8 & 62873.27 & & \\
\hline 1641.874 & 2 & 60906.01 & & & 1590.155 & 80 & 62886.95 & & \\
\hline 1641.623 & 2 & 60915.32 & & & 1589.913 & 50 & 62896.52 & $99165_{13 / 2}$ & $-162061_{11 / 2}^{\circ}$ \\
\hline 1640.948 & 1 & 60940.38 & & & 1589.455 & 60 & 62914.64 & & \\
\hline 1639.060 & 2 & 61010.57 & $117026_{5 / 2}$ & $-178036_{7 / 2}^{\circ}$ & 1589.067 & 80 & 62930.00 & & \\
\hline 1637.952 & 1 & 61051.85 & & & 1588.888 & 1 & 62937.09 & & \\
\hline 1637.809 & 500 & 61057.18 & & & 1588.516 & 1 & 62951.83 & & \\
\hline 1637.627 & 6 & 61063.96 & & & 1588.381 & 30 & 62957.18 & & \\
\hline 1637.525 & 30 & 61067.77 & & & 1588.072 & 60 & 62969.43 & & \\
\hline 1636.479 & 10 & 61106.80 & & & 1587.100 & 600 & 63008.00 & & \\
\hline 1635.607 & 150 & 61139.38 & & & 1586.538 & 1 & 63030.32 & & \\
\hline
\end{tabular}


TABLE 1. Classified spectral lines of $\mathrm{Yb}$ IV.

Wavelengths longer than $2000 \AA$ are values in air-continued

\begin{tabular}{|c|c|c|c|c|c|c|c|c|c|}
\hline $\begin{array}{c}\text { Wavelength } \\
(\AA)\end{array}$ & Intensity & $\begin{array}{c}\text { Wavenumber } \\
\left(\mathrm{cm}^{-1}\right)\end{array}$ & Classific & cation & $\begin{array}{c}\text { Wavelength } \\
(\AA)\end{array}$ & Intensity & $\begin{array}{l}\text { Wavenumber } \\
\left(\mathrm{cm}^{-1}\right)\end{array}$ & \multicolumn{2}{|c|}{ Classification } \\
\hline 1585.606 & 2 & 63067.37 & & & 1561.851 & 2 & 64026.59 & $120328_{5 / 2}$ & $-184355_{5 / 2}^{\circ}$ \\
\hline 1585.230 & 800 & 63082.32 & $122787_{3 / 2}$ & $-185869_{5 / 2}^{\circ}$ & 1561.432 & 8 & 64043.77 & & \\
\hline 1585.200 & 1 & 63083.52 & & & 1561.339 & 100 & 64047.58 & & \\
\hline 1584.926 & 80 & 63094.42 & & & 1559.751 & 20 & 64112.79 & & \\
\hline 1584.882 & 1 & 63096.18 & & & 1558.960 & 30 & 64145.32 & & \\
\hline 1584.824 & 10 & 63098.48 & & & 1556.967 & 20 & 64227.43 & $97592_{9 / 2}$ & $-161820_{9 / 2}$ \\
\hline 1584.496 & 100 & 63111.55 & & & 1555.372 & 2 & 64293.30 & $113049_{11 / 2}$ & $-177343_{9 / 2}^{\circ}$ \\
\hline 1584.371 & 150 & 63116.53 & & & 1556.717 & 200 & 64237.75 & & \\
\hline 1583.181 & 100 & 63163.97 & & & 1555.222 & 40 & 64299.50 & & \\
\hline 1582.729 & 8 & 63182.01 & & & 1554.484 & 4 & 64330.02 & & \\
\hline 1582.438 & 60 & 63193.62 & & & 1554.169 & 10 & 64343.06 & & \\
\hline 1581.901 & 90 & 63215.08 & & & 1553.928 & 2 & 64353.04 & $112189_{9 / 2}$ & $-176542_{7 / 2}^{\circ}$ \\
\hline 1581.856 & 80 & 63216.87 & & & 1553.377 & 6 & 64375.87 & & \\
\hline 1581.760 & 100 & 63220.71 & & & 1552.462 & 10 & 64413.81 & $88175_{11 / 2}$ & $-152589_{11 / 2}$ \\
\hline 1581.403 & 400 & 63234.98 & & & 1551.899 & 50 & 64437.18 & $94498_{5 / 2}$ & $-158935_{7 / 2}^{0}$ \\
\hline 1581.212 & 1 & 63242.62 & & & 1551.576 & 10 & 64450.59 & & \\
\hline 1581.107 & 2 & 63246.82 & & & 1551.126 & 20 & 64469.29 & $97592_{9 / 2}$ & $-16206 \mathrm{l}_{11 / 2}^{\circ}$ \\
\hline 1581.006 & 1 & 63250.86 & & & 1550.774 & 25 & 54483.92 & & \\
\hline 1580.934 & 300 & 63253.74 & & & 1550.256 & 80 & 64505.47 & & \\
\hline 1580.723 & 10 & 63262.19 & & & 1549.876 & 40 & 64521.29 & & \\
\hline 1580.333 & 500 & 63277.80 & $106001_{3 / 2}$ & $-169279_{5 / 2}^{\circ}$ & 1548.896 & 10 & 64562.11 & & \\
\hline 1579.589 & 150 & 63307.60 & & & 1548.201 & 150 & 64591.09 & & \\
\hline 1579.324 & 80 & 63318.22 & & & 1547.465 & 600 & 64621.81 & $127045_{13 / 2}$ & $-191666_{13 / 2}^{\circ}$ \\
\hline 1578.934 & 500 & 63333.86 & & & 1547.283 & 100 & 64629.41 & $106039_{7 / 2}$ & $-170669_{5 / 2}^{2}$ \\
\hline 1578.856 & 100 & 63336.99 & & & 1546.703 & 1 & 64653.65 & $102542_{7 / 2}$ & $-167196_{9 / 2}^{\circ}$ \\
\hline 1578.714 & 1 & 63342.69 & & & 1544.552 & 1 & 64743.69 & & \\
\hline 1578.600 & 100 & 63347.26 & & & 1544.079 & 250 & 64763.52 & $127045_{13 / 2}$ & $-191808_{11 / 2}^{\circ}$ \\
\hline 1578.541 & 40 & 63349.63 & & & 1543.117 & 8 & 64803.90 & $104613_{\mathbf{9} / 2}$ & $-169417_{7 / 2}^{0}$ \\
\hline 1578.123 & 150 & 63366.41 & & & 1542.767 & 10 & 64818.60 & & \\
\hline 1578.051 & 400 & 63369.30 & & & 1541.525 & 200 & 64870.82 & $102542_{7 / 2}$ & $-167413_{7 / 2}^{\circ}$ \\
\hline 1577.860 & 300 & 63376.97 & & & 1538.968 & 18 & 64978.60 & & \\
\hline 1577.453 & 10 & 63393.33 & & & 1537.676 & 20 & 65033.20 & $88175_{11 / 2}$ & $-153208_{13 / 2}^{\varrho}$ \\
\hline 1577.203 & 100 & 63403.37 & & & 1535.698 & 40 & 65116.96 & $112679_{5 / 2}$ & $-177796_{7 / 2}^{\circ}$ \\
\hline 1576.891 & 2 & 63415.92 & & & 1532.442 & 10 & 65255.32 & $102158_{5 / 2}$ & $-167413_{7 / 2}^{\circ}$ \\
\hline 1576.757 & 40 & 63421.31 & & & 1532.160 & 20 & 65267.33 & & \\
\hline 1576.587 & 50 & 63428.15 & & & 1530.050 & 30 & 65357.34 & $112679_{5 / 2}$ & $-178036_{7 / 2}^{\circ}$ \\
\hline 1576.564 & 200 & 63429.07 & $113049_{11 / 2}$ & $-176478_{9 / 2}^{\circ}$ & 1529.739 & 1 & 65370.62 & $112679_{5 / 2}$ & $-178049_{5 / 2}$ \\
\hline 1576.032 & 50 & 63450.48 & & & 1529.273 & 1 & 65390.54 & & \\
\hline 1575.767 & 30 & 63461.15 & & & 1529.078 & 150 & 65398.88 & $97592_{9 / 2}$ & $-162991_{9 / 2}^{\circ}$ \\
\hline 1574.974 & 2000 & 63493.11 & & & 1529.009 & 5 & 65401.83 & & \\
\hline 1574.892 & 8 & 63496.41 & & & 1528.952 & 1 & 65404.27 & $97587_{11 / 2}$ & $-16299 \mathrm{l}_{9 / 2}^{\circ}$ \\
\hline 1574.715 & 150 & 63503.55 & & & 1528.826 & 2 & 65409.66 & $\left\{106801_{11 / 2}\right.$ & $-17221 l_{11 / 2}^{\circ}$ \\
\hline 1574.571 & 200 & 63509.36 & & & & & & \{ 97592//2 & $-163002_{11 / 2}^{9}$ \\
\hline 1574.301 & 10 & 63520.25 & & & 1528.620 & 35 & 65418.48 & & \\
\hline 1573.839 & 200 & 63538.90 & & & 1528.528 & 2 & 65422.41 & & \\
\hline 1573.569 & 60 & 63549.80 & & & 1527.961 & 8 & 65446.69 & $101966_{9 / 2}$ & $-167413_{7 / 2}^{\circ}$ \\
\hline 1573.379 & 30 & 63557.47 & & & 1527.410 & 10 & 65470.30 & $106039_{7 / 2}$ & $-171510_{7 / 2}$ \\
\hline 1573.205 & 50 & 63564.50 & & & 1524.804 & 2 & 65582.19 & $102542_{7 / 2}$ & $-168124_{7 / 2}^{\circ}$ \\
\hline 1572.882 & 1 & 63577.56 & & & 1524.607 & 40 & 65590.67 & & \\
\hline 1572.688 & 1 & 63585.40 & & & 1523.518 & 80 & 65637.55 & $118717_{7 / 2}$ & $-184355_{5 / 2}^{\circ}$ \\
\hline 1572.463 & 100 & 63594.50 & $105685_{7 / 2}$ & $-169279_{5 / 2}^{\circ}$ & 1523.351 & 40 & 65644.75 & & \\
\hline 1571.534 & 40 & 63632.09 & & & 1523.234 & 20 & 65649.79 & & \\
\hline 1571.410 & 10 & 63637.11 & $107873_{5 / 2}$ & $-171510_{7 / 2}^{\circ}$ & 1521.468 & 15 & 65725.99 & & \\
\hline 1571.270 & 20 & 63642.78 & & & 1521.346 & 100 & 65731.26 & & \\
\hline 1571.069 & 10 & 63650.92 & & & 1519.908 & 30 & 65793.45 & $95316_{13 / 2}$ & $-161110_{15 / 2}^{\circ}$ \\
\hline 1570.944 & 30 & 63655.99 & & & 1519.785 & 60 & 65798.78 & & \\
\hline 1570.827 & 20 & 63660.73 & & & 1519.558 & 1 & 65808.60 & & \\
\hline 1570.574 & 150 & 63670.98 & & & 1510.980 & 2 & 66182.21 & $105685_{7 / 2}$ & $-171867_{\mathbf{9} / 2}^{\circ}$ \\
\hline 1570.288 & 20 & 63682.58 & & & 1509.466 & 10 & 66248.59 & & \\
\hline 1570.104 & 10 & 63690.04 & & & 1505.035 & 50 & 66443.63 & $104225_{7 / 2}$ & $-170669_{5 / 2}^{\circ}$ \\
\hline 1569.068 & 100 & 63732.10 & $10600 \mathrm{l}_{3 / 2}$ & $-169733_{3 / 2}^{\circ}$ & 1502.975 & 20 & 66534.70 & $110808_{7 / 2}$ & $-177343_{9 / 2}^{\circ / 2}$ \\
\hline 1568.742 & 30 & 63745.34 & & & 1499.165 & 20 & 66703.79 & & \\
\hline 1568.296 & 20 & 63763.47 & & & 1498.409 & 10 & 66737.45 & $102542_{7 / 2}$ & $-169279_{5 / 2}^{\circ}$ \\
\hline 1567.745 & 4 & 63785.88 & $89422_{13 / 2}$ & $-153208_{13 / 2}^{\circ}$ & 1498.242 & 10 & 66744.89 & $95316_{13 / 2}^{7 / 2}$ & $-162061_{11 / 2}^{5 / 2}$ \\
\hline 1567.452 & 1 & 63797.80 & & & 1497.742 & 80 & 66767.17 & $102512_{3 / 2}$ & $-169279_{5 / 2}^{\circ}$ \\
\hline 1567.192 & 300 & 63808.39 & & & 1497.684 & 80 & 66769.75 & $124897_{11 / 2}^{3 / 2}$ & $-191666_{13 / 2}^{\circ / 2}$ \\
\hline 1566.494 & 10 & 63836.82 & & & 1495.323 & 80 & 66875.18 & $102542_{7 / 2}$ & $-169417_{7 / 2}^{\circ}$ \\
\hline 1565.888 & 60 & 63861.52 & $95078_{11 / 2}$ & $-158939_{9 / 2}^{\circ}$ & 1494.830 & 2 & 66897.23 & $104613_{9 / 2}$ & $-171510_{7 / 2}^{\circ}$ \\
\hline 1565.755 & 1 & 63866.95 & $104225_{7 / 2}$ & $-168092_{9 / 2}^{0 / 2}$ & 1494.506 & 500 & 66911.74 & $124897_{11 / 2}^{3 / 2}$ & $-191808_{11 / 2}^{\circ}$ \\
\hline 1564.246 & 1 & 63928.56 & & & 1494.029 & 100 & 66933.10 & & \\
\hline 1564.059 & 100 & 63936.20 & & & 1492.894 & 500 & 66983.99 & $95078_{11 / 2}$ & $-162061_{11 / 2}^{\circ}$ \\
\hline 1563.481 & 2 & 53959.84 & & & 1492.811 & 300 & 66987.71 & $110808_{7 / 2}$ & $-177796_{7 / 2}^{\circ}$ \\
\hline 1562.906 & 1 & 63983.37 & & & 1491.566 & 3000 & 67043.63 & $85545_{13 / 2}$ & $-152589_{11 / 2}^{\circ}$ \\
\hline 1562.642 & 80 & 63994.18 & & & 1489.827 & 60 & 67121.88 & $102158_{5 / 2}$ & $-169279_{5 / 2}^{\circ}$ \\
\hline
\end{tabular}


TABLE 1. Classified spectral lines of $\mathrm{Yb}$ IV.

Wavelengths longer than $2000 \AA$ are values in air-continued

\begin{tabular}{|c|c|c|c|c|c|c|c|c|c|}
\hline $\begin{array}{c}\text { Wavelength } \\
(\AA)\end{array}$ & Intensity & $\begin{array}{l}\text { Wavenumber } \\
\left(\mathrm{cm}^{-1}\right)\end{array}$ & \multicolumn{2}{|c|}{ Classification } & $\begin{array}{c}\text { Wavelength } \\
(\AA)\end{array}$ & Intensity & $\begin{array}{l}\text { Wavenumber } \\
\left(\mathrm{cm}^{-1}\right)\end{array}$ & \multicolumn{2}{|c|}{ Classification } \\
\hline 1489.163 & 1 & 67151.81 & $118717_{7 / 2}$ & $-185869_{5 / 2}^{\circ}$ & 1423.275 & 20 & 70260.49 & & \\
\hline 1487.693 & 50 & 67218.16 & & & 1422.871 & 40 & 70280.43 & $109025_{9 / 2}$ & $-179305_{7 / 2}^{\circ}$ \\
\hline 1487.640 & 15 & 67220.56 & $102512_{3 / 2}$ & $-169733_{3 / 2}^{\circ}$ & 1422.088 & 800 & 70319.13 & $100350_{7 / 2}$ & $-170669_{5 / 2}^{\circ}$ \\
\hline 1487.473 & 30 & 67228.11 & $110808_{7 / 2}$ & $-178036_{7 / 2}^{\circ}$ & 1421.627 & 1 & 70341.93 & & \\
\hline 1487.196 & 2 & 67240.63 & $100350_{7 / 2}$ & $-167590_{5 / 2}^{\circ}$ & 1421.383 & 1 & 70354.01 & & \\
\hline 1486.906 & 20 & 67253.74 & $104613_{9 / 2}$ & $-171867_{9 / 2}^{\circ}$ & 1420.885 & 150 & 70378.67 & & \\
\hline 1486.883 & 40 & 67254.78 & $118717_{7 / 2}$ & $-185972_{7 / 2}^{\circ}$ & 1420.688 & 200 & 70388.43 & $99344_{5 / 2}$ & $-169733_{3 / 2}^{\circ}$ \\
\hline 1486.777 & 15 & 67259.58 & $102158_{5 / 2}$ & $-169417_{7 / 2}^{\circ}$ & 1419.669 & 50 & 70438.95 & $106039_{7 / 2}$ & $-176478_{9 / 2}^{\circ}$ \\
\hline 1485.483 & 600 & 67318.17 & $95316_{13 / 2}$ & $-162635_{13 / 2}^{\circ}$ & 1419.633 & 60 & 70440.74 & & \\
\hline 1485.238 & 100 & 67329.27 & $117026_{5 / 2}$ & $-184355_{5 / 2}^{\circ}$ & 1419.530 & 250 & 70445.85 & $9897 l_{9 / 2}$ & $-169417_{7 / 2}^{\circ}$ \\
\hline 1484.285 & 3 & 67372.50 & & & 1419.261 & 2 & 70459.20 & & \\
\hline 1483.755 & 1 & 67396.57 & & & 1418.386 & 50 & 70502.66 & $106039_{7 / 2}$ & $-176542_{7 / 2}^{\circ}$ \\
\hline 1483.605 & 2 & 67403.38 & $91532_{7 / 2}$ & $-158935_{7 / 2}^{\circ}$ & 1417.962 & 1 & 70523.75 & & \\
\hline 1482.553 & 400 & 67451.21 & $101966_{9 / 2}$ & $-169417_{7 / 2}^{\circ}$ & 1417.761 & 350 & 70533.74 & & \\
\hline 1482.506 & 300 & 67453.35 & $\left\{91482_{5 / 2}^{5 / 2}\right.$ & $-158935_{7 / 2}^{\circ}$ & 1417.719 & 4000 & 70535.84 & $82673_{11 / 2}$ & $-153208_{13 / 2}^{\circ}$ \\
\hline & & & $\left\{109025_{9 / 2}\right.$ & $-176478_{9 / 2}^{\circ}$ & 1417.617 & 250 & 70540.91 & $10680 l_{11 / 2}$ & $-177343_{9 / 2}^{\circ}$ \\
\hline 1477.916 & 4000 & 67662.84 & $85545_{13 / 2}$ & $-153208_{13 / 2}^{\circ}$ & 1417.111 & 6 & 70566.10 & & \\
\hline 1474.744 & 1500 & 67808.37 & & & 1416.814 & 30 & 70580.89 & & \\
\hline 1474.513 & 800 & 67819.00 & & & 1416.386 & 1 & 70602.22 & & \\
\hline 1472.457 & 800 & 67913.69 & $95078_{11 / 2}$ & $-162991_{9 / 2}^{\circ}$ & 1416.219 & 8 & 70610.54 & & \\
\hline 1472.228 & 1000 & 67924.26 & $95078_{11 / 2}$ & $-163002_{11 / 2}^{\circ}$ & 1416.153 & 4000 & 70613.83 & $88322_{5 / 2}$ & $-158935_{7 / 2}^{\circ}$ \\
\hline 1471.420 & 2 & 67961.56 & $101318_{5 / 2}$ & $-169279_{5 / 2}^{\circ}$ & 1416.108 & 200 & 70616.08 & & \\
\hline 1469.109 & 200 & 68068.46 & $99344_{5 / 2}$ & $-167413_{7 / 2}^{\circ}$ & 1415.642 & 1 & 70639.32 & & \\
\hline 1468.321 & 1000 & 68104.99 & $90834_{11 / 2}$ & $-158939_{9 / 2}^{\circ}$ & 1414.358 & 300 & 70703.45 & $91931_{13 / 2}$ & $-162635_{13 / 2}^{\circ}$ \\
\hline 1466.508 & 400 & 68189.19 & & & 1413.645 & 1 & 70739.11 & & \\
\hline 1465.752 & 800 & 68224.36 & $98971_{9 / 2}$ & $-167196_{9 / 2}^{\circ}$ & 1413.144 & 3000 & 70764.19 & $88175_{11 / 2}$ & $-158939_{9 / 2}^{\circ}$ \\
\hline 1465.244 & 3 & 68248.01 & & & 1412.881 & 600 & 70777.36 & $98502_{7 / 2}$ & $-169279_{5 / 2}^{\circ}$ \\
\hline 1463.744 & 800 & 68317.95 & $109025_{9 / 2}$ & $-177343_{9 / 2}^{\circ}$ & 1412.708 & 200 & 70786.03 & $106557_{11 / 2}$ & $-177343_{9 / 2}^{\circ}$ \\
\hline 1462.624 & 150 & 68370.27 & $115984_{7 / 2}^{9 / 2}$ & $-184355_{5 / 2}^{\circ / 2}$ & 1412.200 & 500 & 70811.49 & $107873_{5 / 2}$ & $-178684_{5 / 2}^{\mathrm{g} / 2}$ \\
\hline 1462.357 & 200 & 68382.75 & $109413_{5 / 2}$ & $-177796_{7 / 2}^{\circ}$ & 1412.003 & 1000 & 70821.37 & $88118_{9 / 2}$ & $-158939_{9 / 2}^{8 / 2}$ \\
\hline 1461.848 & 100 & 68406.56 & & & 1411.599 & 40 & 70841.64 & & \\
\hline 1461.103 & 600 & 68441.44 & $98971_{9 / 2}$ & $-167413_{7 / 2}^{\circ}$ & 1410.814 & 20 & 70881.06 & & \\
\hline 1459.614 & 20 & 68511.26 & $102158_{5 / 2}$ & $-170669_{5 / 2}^{\circ}$ & 1410.458 & 300 & 70898.95 & & \\
\hline 1457.236 & 3 & 68623.06 & $109413_{5 / 2}$ & $-178036_{7 / 2}^{\circ / 2}$ & 1410.252 & 1 & 70909.31 & & \\
\hline 1457.049 & 150 & 68631.87 & & & 1410.133 & 2 & 70915.29 & $98502_{7 / 2}$ & $-169417_{7 / 2}^{\circ}$ \\
\hline 1455.526 & 4 & 68703.68 & & & 1409.421 & 1 & 70951.12 & & \\
\hline 1454.778 & 3 & 68739.01 & $93081_{11 / 2}$ & $-161820_{9 / 2}^{\circ}$ & 1408.799 & 2 & 70982.44 & & \\
\hline 1454.100 & 100 & 68771.06 & $109025_{9 / 2}^{11 / 2}$ & $-177796_{7 / 2}^{\circ / 2}$ & 1408.733 & 800 & 70985.77 & $90834_{11 / 2}$ & $-161820_{9 / 2}^{\circ}$ \\
\hline 1453.913 & 100 & 68779.90 & $99344_{5 / 2}$ & $-168124_{7 / 2}^{\circ}$ & 1408.579 & 60 & 70993.53 & & \\
\hline 1452.310 & 4 & 68855.82 & $99165_{13 / 2}$ & $-16802 l_{11 / 2}^{\circ}$ & 1408.374 & 80 & 71003.86 & $130889_{13 / 2}$ & $-201893_{11 / 2}^{\circ}$ \\
\hline 1451.990 & 300 & 68870.99 & $130855_{15 / 2}$ & $-199726_{15 / 2}^{\circ}$ & 1408.248 & 1 & 71010.22 & & \\
\hline 1451.593 & 1000 & 68889.83 & $90045_{9 / 2}$ & $-158935_{7 / 2}^{\circ}$ & 1407.325 & 200 & 71056.79 & $98222_{5 / 2}$ & $-169279_{5 / 2}^{\circ}$ \\
\hline 1451.144 & 80 & 68911.14 & $98502_{7 / 2}$ & $-167413_{7 / 2}^{\circ}$ & 1407.256 & 80 & 71060.27 & & \\
\hline 1451.118 & 80 & 68912.38 & $\left\{99180_{11 / 2}\right.$ & $-168092_{9 / 2}^{\circ}$ & 1407.049 & 4000 & 71070.73 & $91931_{13 / 2}$ & $-163002_{11 / 2}^{\circ}$ \\
\hline & & & $\left\{107566_{9 / 2}\right.$ & $-176478_{9 / 2}^{\circ}$ & 1406.550 & 3 & 71095.94 & & \\
\hline 1450.841 & 800 & 68925.54 & & & 1406.206 & 10 & 71113.33 & & \\
\hline 1449.679 & 3 & 68980.78 & $93081_{11 / 2}$ & $-162061_{11 / 2}^{\circ}$ & 1405.278 & 4 & 71160.29 & $100350_{7 / 2}$ & $-171510_{7 / 2}^{\circ}$ \\
\hline 1449.036 & 2 & 69011.39 & $109025_{9 / 2}$ & $-178036_{7 / 2}^{\circ}$ & 1404.778 & 200 & 71185.62 & & \\
\hline 1445.290 & 30 & 69190.26 & $98222_{5 / 2}$ & $-167413_{7 / 2}^{\circ}$ & 1404.589 & 100 & 71195.20 & $98222_{5 / 2}$ & $-169417_{7 / 2}^{\circ}$ \\
\hline 1442.732 & 6 & 69312.94 & & & 1404.116 & 80 & 71219.18 & $95977_{7 / 2}^{5 / 2}$ & $-167196_{9 / 2}^{\circ}$ \\
\hline 1441.933 & 50 & 69351.35 & $101318_{5 / 2}$ & $-170669_{5 / 2}^{\circ}$ & 1403.950 & 1000 & 71227.60 & $90834_{11 / 2}$ & $-162061_{11 / 2}^{\circ / 2}$ \\
\hline 1441.735 & 4 & 69360.87 & $102850_{11 / 2}$ & $-172211_{11 / 2}^{\circ}$ & 1403.717 & 300 & 71239.43 & & \\
\hline 1440.862 & 50 & 69402.89 & & & 1402.452 & 5 & 71303.68 & & \\
\hline 1440.609 & 2000 & 69415.08 & $89520_{7 / 2}$ & $-158935_{7 / 2}^{\circ}$ & 1402.033 & 60 & 71324.99 & $99344_{5 / 2}$ & $-170669_{5 / 2}^{\circ}$ \\
\hline 1440.391 & 20 & 69425.59 & & & 1401.919 & 10 & 71330.79 & & \\
\hline 1439.867 & 30 & 69450.85 & & & 1401.650 & 800 & 71344.48 & & \\
\hline 1437.943 & 800 & 69543.78 & $101966_{9 / 2}$ & $-171510_{7 / 2}^{\circ}$ & 1401.087 & 1 & 71373.15 & & \\
\hline 1437.364 & 20 & 69571.79 & & & 1400.419 & 300 & 71407.19 & & \\
\hline 1436.691 & 100 & 69604.39 & & & 1400.365 & 250 & 71409.95 & $130889_{13 / 2}$ & $-202299_{13 / 2}^{\circ}$ \\
\hline 1436.621 & 1000 & 69607.78 & $97587_{11 / 2}$ & $-167196_{9 / 2}^{\circ}$ & 1399.846 & 600 & 71436.43 & $95977_{7 / 2}^{13 / 2}$ & $-167413_{7 / 2}^{\circ}$ \\
\hline 1434.913 & 1 & 69690.63 & & & 1399.702 & 300 & 71443.77 & $130855_{15 / 2}$ & $-202299_{13 / 2}^{\circ}$ \\
\hline 1432.894 & 100 & 69788.83 & & & 1398.819 & 17 & 71488.87 & & \\
\hline 1430.935 & 2 & 69884.37 & $115984_{7 / 2}$ & $-185869_{5 / 2}^{\circ}$ & 1398.773 & 3500 & 71491.22 & & \\
\hline 1430.609 & 1500 & 69900.30 & $101966_{9 / 2}$ & $-171867_{9 / 2}^{\circ}$ & 1398.393 & 2 & 71510.65 & $98222_{5 / 2}$ & $-169733_{3 / 2}^{\circ}$ \\
\hline 1430.391 & 30 & 69910.95 & $9308 l_{11 / 2}$ & $-162991_{9 / 2}^{\circ}$ & 1398.323 & 1 & 71514.23 & & \\
\hline 1430.294 & 3000 & 69915.69 & $82673_{11 / 2}$ & $-152589_{11 / 2}^{\circ}$ & 1398.298 & 15 & 71515.51 & & \\
\hline 1429.900 & 500 & 69934.96 & $99344_{5 / 2}$ & $-169279_{5 / 2}^{\circ}$ & 1397.837 & 30 & 71539.09 & $104225_{7 / 2}$ & $-175765_{5 / 2}^{\circ}$ \\
\hline 1427.868 & 80 & 70034.48 & & & 1396.518 & 1 & 71606.66 & & \\
\hline 1427.094 & 30 & 70072.46 & $99344_{5 / 2}$ & $-169417_{7 / 2}^{\circ}$ & 1396.375 & 1 & 71614.00 & $95977_{7 / 2}$ & $-167590_{5 / 2}^{\circ}$ \\
\hline 1426.258 & 10 & 70113.54 & & & 1395.159 & 2 & 71676.41 & $112679_{5 / 2}$ & $-184355_{5 / 2}^{\circ}$ \\
\hline 1425.925 & 800 & 70129.91 & $91931_{13 / 2}$ & $-162061_{11 / 2}^{\circ}$ & 1394.934 & 30 & 71687.97 & $89422_{13 / 2}$ & $-161110_{15 / 2}^{\circ / 2}$ \\
\hline 1424.662 & 60 & 70192.08 & $101318_{5 / 2}$ & $-171510_{7 / 2}^{\circ}$ & 1394.577 & 10 & 71706.33 & & \\
\hline 1423.991 & 2000 & 70225.16 & & & 1394.075 & 4 & 71732.15 & & \\
\hline 1423.894 & 80 & 70229.94 & $107505_{9 / 2}$ & $-177796_{7 / 2}^{\circ}$ & 1393.931 & 2500 & 71739.56 & $107566_{9 / 2}$ & $-179305_{7 / 2}^{\circ}$ \\
\hline
\end{tabular}


TABLE 1. Classified spectral lines of Yb IV.

Wavelengths longer than $2000 \AA$ are values in air-continued

\begin{tabular}{|c|c|c|c|c|c|c|c|c|c|}
\hline $\begin{array}{c}\text { Wavelength } \\
(\AA)\end{array}$ & Intensity & $\begin{array}{l}\text { Wavenumber } \\
\left(\mathrm{cm}^{-1}\right)\end{array}$ & \multicolumn{2}{|c|}{ Classification } & $\begin{array}{l}\text { Wavelength } \\
(\AA)\end{array}$ & Intensity & $\begin{array}{c}\text { Wavenumber } \\
\left(\mathrm{cm}^{-1}\right)\end{array}$ & \multicolumn{2}{|c|}{ Classification } \\
\hline 1393.874 & 600 & 71742.49 & & & 1364.203 & 30 & 73302.87 & $95977_{7 / 2}$ & $-169279_{5 / 2}^{\circ}$ \\
\hline 1393.526 & 250 & 71760.41 & & & 1363.053 & 2 & 73364.71 & $98502_{7 / 2}$ & $-171867_{9 / 2}^{\circ}$ \\
\hline 1393.332 & 2 & 71770.40 & & & 1361.966 & 800 & 73423.27 & $104613_{\mathbf{9} / \mathbf{2}}$ & $-178036_{7 / 2}^{\circ}$ \\
\hline 1392.981 & 40 & 71788.48 & $112679_{5 / 2}$ & $-184467_{3 / 2}^{\circ}$ & 1361.746 & 2000 & 73435.13 & $118231_{15 / 2}$ & $-191666_{13 / 2}^{\circ}$ \\
\hline 1392.954 & 1 & 71789.88 & & & 1361.647 & 100 & 73440.47 & $95977_{7 / 2}$ & $-169417_{7 / 2}^{0}$ \\
\hline 1392.883 & 400 & 71793.53 & $99180_{11 / 2}$ & $-170974_{13 / 2}^{\circ}$ & 1360.965 & 40 & 73477.27 & & \\
\hline 1392.727 & 4 & 71801.58 & & & 1359.833 & 800 & 73538.44 & & \\
\hline 1392.646 & 200 & 71805.75 & & & 1359.680 & 1 & 73546.71 & $110808_{7 / 2}$ & $-184355_{5 / 2}^{\circ}$ \\
\hline 1392.619 & 1500 & 71807.14 & & & 1359.064 & 1 & 73580.05 & $89422_{13 / 2}$ & $-163002_{11 / 2}$ \\
\hline 1392.580 & 1000 & 71809.15 & $99165_{13 / 2}$ & $-170974_{13 / 2}^{\circ}$ & 1358.656 & 80 & 73602.14 & & \\
\hline 1392.404 & 60 & 71818.23 & & & 1358.628 & 1000 & 73603.66 & $102875_{9 / 2}$ & $-176478_{\mathbf{9} / 2}^{\circ}$ \\
\hline 1391.728 & 1 & 71853.12 & & & 1358.318 & 1 & 73620.46 & $105685_{7 / 2}$ & $-179305_{7 / 2}^{\circ}$ \\
\hline 1391.483 & 2 & 71865.77 & $104613_{9 / 2}$ & $-176478_{9 / 2}^{\circ}$ & 1358.175 & 200 & 73628.21 & $102850_{11 / 2}$ & $-176478_{9 / 2}^{\circ}$ \\
\hline 1390.248 & 10 & 71929.61 & $104613_{9 / 2}$ & $-176542_{7 / 2}^{\circ}$ & 1357.783 & 250 & 73649.47 & $112812_{9 / 2}$ & $-186462_{9 / 2}^{\circ}$ \\
\hline 1388.948 & 10 & 71996.93 & $106039_{7 / 2}$ & $-178036_{7 / 2}^{\circ}$ & 1357.472 & 200 & 73666.34 & & \\
\hline 1388.629 & 100 & 72013.47 & & & 1357.156 & 30 & 73683.49 & & \\
\hline 1387.957 & 450 & 72048.34 & $106001_{3 / 2}$ & $-178049_{5 / 2}^{\circ}$ & 1357.057 & 500 & 73688.87 & $112772_{7 / 2}$ & $-186462_{9 / 2}^{\circ}$ \\
\hline 1387.714 & 200 & 72060.95 & & & 1356.818 & 1000 & 73701.85 & $88118_{9 / 2}$ & $-161820_{9 / 2}^{\circ}$ \\
\hline 1387.385 & 40 & 72078.04 & & & 1356.146 & 4000 & 73738.37 & $94386_{9 / 2}$ & $-168124_{7 / 2}^{0}$ \\
\hline 1387.334 & 800 & 72080.69 & & & 1354.811 & 800 & 73811.03 & $104225_{7 / 2}$ & $-178036_{7 / 2}^{\circ}$ \\
\hline 1385.913 & 60 & 72154.60 & & & 1354.697 & 30 & 73817.24 & & \\
\hline 1385.847 & 20 & 72158.03 & $90834_{11 / 2}$ & $-162991_{9 / 2}^{\circ}$ & 1354.578 & 10 & 73823.72 & $104225_{7 / 2}$ & $-178049_{5 / 2}^{\circ}$ \\
\hline 1385.678 & 2 & 72166.83 & $98502_{7 / 2}$ & $-170669_{5 / 2}^{\circ}$ & 1353.428 & 2000 & 73886.45 & $88175_{11 / 2}$ & $-16206 l_{11 / 2}^{\circ}$ \\
\hline 1385.643 & 30 & 72168.66 & $90834_{11 / 2}$ & $-163002_{11 / 2}^{\circ}$ & 1352.973 & 80 & 73911.30 & $104613_{9 / 2}$ & $-178524_{9 / 2}^{\circ}$ \\
\hline 1385.349 & 30 & 72183.97 & & & 1352.383 & 1000 & 73943.55 & $88118_{9 / 2}$ & $-16206 l_{11 / 2}^{\circ}$ \\
\hline 1385.067 & 30 & 72198.67 & & & 1352.149 & 200 & 73956.34 & $118717_{7 / 2}$ & $-192674_{5 / 2}^{0}$ \\
\hline 1384.406 & 2000 & 72233.14 & $88877_{15 / 2}$ & $-161110_{15 / 2}^{\circ}$ & 1350.264 & 9000 & 74059.59 & $78529_{9 / 2}$ & $-152589_{11 / 2}^{\circ}$ \\
\hline 1384.189 & 30 & 72244.47 & & & 1350.176 & 20 & 74064.41 & & \\
\hline 1382.852 & 20 & 72314.31 & & & 1349.829 & 1500 & 74083.45 & & \\
\hline 1382.801 & 50 & 72316.98 & $104225_{7 / 2}$ & $-176542_{7 / 2}^{\circ}$ & 1348.925 & 1000 & 74133.10 & $10214_{5 / 2}^{\circ}$ & $-\quad 84347_{7 / 2}$ \\
\hline 1382.519 & 1000 & 72331.73 & & & 1348.658 & 2 & 74147.78 & & \\
\hline 1382.255 & 40 & 72345.55 & $120328_{5 / 2}$ & $-192674_{5 / 2}^{\circ}$ & 1347.668 & 300 & 74202.25 & $95215_{9 / 2}$ & $-169417_{7 / 2}^{\circ}$ \\
\hline 1381.930 & 80 & 72362.56 & $94834_{7 / 2}$ & $-167196_{9 / 2}^{\circ}$ & 1346.939 & 600 & 74242.41 & & \\
\hline 1381.881 & 60 & 72365.13 & $105685_{7 / 2}$ & $-178049_{5 / 2}^{\circ}$ & 1345.815 & 4 & 74304.41 & & \\
\hline 1381.500 & 50 & 72385.08 & & & 1345.675 & 100 & 74312.14 & $103484_{5 / 2}$ & $-177796_{7 / 2}^{\circ}$ \\
\hline 1381.179 & 150 & 72401.91 & & & 1345.558 & 20 & 74318.60 & $111550_{7 / 2}$ & $-185869_{5 / 2}^{0}$ \\
\hline 1380.372 & 150 & 72444.23 & & & 1345.362 & 3000 & 74329.43 & & \\
\hline 1379.985 & 100 & 72464.55 & & & 1344.574 & 1000 & 74372.99 & & \\
\hline 1379.287 & 1 & 72501.22 & & & 1344.467 & 1000 & 74378.91 & & \\
\hline 1379.017 & 100 & 72515.42 & & & 1344.365 & 250 & 74384.56 & $102158_{5 / 2}$ & $-176542_{7 / 2}^{\circ}$ \\
\hline 1378.877 & 80 & 72522.78 & & & 1343.694 & 600 & 74421.70 & $111550_{7 / 2}$ & $-185972_{7 / 2}^{\circ}$ \\
\hline 1376.826 & 1000 & 72630.81 & & & 1343.242 & 150 & 74446.74 & $101318_{5 / 2}$ & $-175765_{5 / 2}^{\circ}$ \\
\hline 1376.722 & 1000 & 72636.30 & $120773_{9 / 2}$ & $-193409_{7 / 2}^{\circ}$ & 1343.047 & 1000 & 74457.55 & & \\
\hline 1376.664 & 3000 & 72639.36 & $89422_{13 / 2}$ & $-16206 \mathrm{l}_{11 / 2}^{\circ}$ & 1343.008 & 800 & 74459.72 & $88175_{11 / 2}$ & $-162635_{13 / 2}^{\circ}$ \\
\hline 1376.556 & 80 & 72645.06 & $106039_{7 / 2}$ & $-178684_{5 / 2}^{\circ}$ & 1342.866 & 40 & 74467.59 & $102875_{9 / 2}$ & $-177343_{9 / 2}^{\circ}$ \\
\hline 1375.838 & 400 & 72682.97 & $106001_{3 / 2}$ & $-178684_{5 / 2}^{\circ}$ & 1342.693 & 1 & 74477.18 & & \\
\hline 1375.417 & 4000 & 72705.22 & $95316_{13 / 2}$ & $-168021_{11 / 2}^{\circ}$ & 1342.188 & 300 & 74505.21 & & \\
\hline 1375.022 & 30 & 72726.10 & & & 1341.329 & 50 & 74552.92 & $103484_{5 / 2}$ & $-178036_{7 / 2}^{\circ}$ \\
\hline 1374.438 & 600 & 72757.01 & $94834_{7 / 2}$ & $-167590_{5 / 2}^{\circ}$ & 1341.102 & 600 & 74565.54 & $103484_{5 / 2}^{5 / 2}$ & $-178049_{5 / 2}^{\circ}$ \\
\hline 1373.524 & 40 & 72805.42 & $95215_{9 / 2}$ & $-168021_{11 / 2}^{0}$ & 1340.930 & 1000 & 74575.10 & & \\
\hline 1373.448 & 800 & 72809.45 & $94386_{9 / 2}$ & $-167196_{9 / 2}^{\circ}$ & 1340.188 & 1 & 74616.39 & & \\
\hline 1372.706 & 20 & 72848.81 & & & 1340.150 & 200 & 74618.51 & $97592_{9 / 2}$ & $-172211_{11 / 2}^{\circ}$ \\
\hline 1371.820 & 2 & 72895.86 & $98971_{9 / 2}$ & $-171867_{9 / 2}^{\circ}$ & 1340.055 & 2000 & 74623.80 & $97587_{11 / 2}$ & $-172211_{11 / 2}^{0}$ \\
\hline 1371.450 & 600 & 72915.52 & $94498_{5 / 2}$ & $-167413_{7 / 2}^{\circ / 2}$ & 1338.834 & 60 & 74691.85 & $\left\{104613_{9 / 2}\right.$ & $-179305_{7 / 2}^{\circ}$ \\
\hline 1370.166 & 400 & 72983.85 & & & & & & $\left\{118717_{7 / 2}\right.$ & $-193409_{7 / 2}^{\circ}$ \\
\hline 1369.884 & 200 & 72998.88 & & & 1337.235 & 2 & 74781.17 & $94498_{5 / 2}$ & $-169279_{5 / 2}^{0}$ \\
\hline 1369.871 & 800 & 72999.57 & $105685_{7 / 2}$ & $-178684_{5 / 2}^{\circ}$ & 1336.414 & 1000 & 74827.11 & $88175_{11 / 2}$ & $-163002_{11 / 2}^{\circ}$ \\
\hline 1369.717 & 3000 & 73007.78 & $98502_{7 / 2}$ & $-171510_{7 / 2}^{\circ}$ & 1336.044 & 2 & 74847.83 & $127045_{13 / 2}$ & $-201893_{11 / 2}^{0}$ \\
\hline 1369.579 & 200 & 73015.13 & $95078_{11 / 2}$ & $-168092_{9 / 2}^{\circ}$ & 1335.390 & 450 & 74884.49 & $88118_{9 / 2}$ & $-163002_{11 / 2}^{\circ}$ \\
\hline 1369.282 & 30 & 73030.97 & $99180_{11 / 2}$ & $-17221 l_{11 / 2}^{\circ}$ & 1334.921 & 600 & 74910.79 & $111550_{7 / 2}$ & $-186462_{9 / 2}^{\circ}$ \\
\hline 1368.129 & 600 & 73092.52 & $94498_{5 / 2}$ & $-167590_{5 / 2}^{\circ}$ & 1334.775 & 1 & 74918.99 & $94498_{5 / 2}$ & $-169417_{7 / 2}^{0 / 2}$ \\
\hline 1367.682 & 300 & 73116.41 & & & 1334.389 & 1000 & 74940.66 & $93081_{11 / 2}$ & $-16802 l_{11 / 2}^{0}$ \\
\hline 1367.307 & 3 & 73136.46 & & & 1333.341 & 3 & 74999.56 & & \\
\hline 1366.874 & 400 & 73159.63 & $112812_{9 / 2}$ & $-185972_{7 / 2}^{\circ}$ & 1333.119 & 1000 & 75012.05 & $93081_{11 / 2}$ & $-168092_{9 / 2}^{\circ}$ \\
\hline 1366.436 & 80 & 73183.08 & $104613_{\mathbf{9} / 2}$ & $-177796_{7 / 2}^{\circ}$ & 1332.796 & 20 & 75030.23 & $94386_{9 / 2}$ & $-169417_{7 / 2}^{\circ}$ \\
\hline 1366.304 & 100 & 73190.15 & $112679_{5 / 2}$ & $-185869_{5 / 2}^{\circ}$ & 1331.926 & 80 & 75079.24 & $104225_{7 / 2}$ & $-179305_{7 / 2}^{\circ}$ \\
\hline 1366.227 & 150 & 73194.27 & $112675_{3 / 2}$ & $-185869_{5 / 2}^{\circ}$ & 1329.780 & 1 & 75200.40 & $103484_{5 / 2}$ & $-178684_{5 / 2}^{\circ}$ \\
\hline 1365.885 & 3000 & 73212.60 & $89422_{13 / 2}$ & $-162635_{13 / 2}^{\circ}$ & 1329.357 & 80 & 75224.33 & $101318_{5 / 2}$ & $-176542_{7 / 2}^{\circ}$ \\
\hline 1365.696 & 30 & 73222.73 & $102542_{7 / 2}$ & $-175765_{5 / 2}^{\circ}$ & 1329.173 & 20 & 75234.75 & $94498_{5 / 2}$ & $-169733_{3 / 2}^{\circ}$ \\
\hline 1365.331 & 1 & 73242.31 & & & 1328.965 & 80 & 75246.52 & & \\
\hline 1365.139 & 200 & 73252.61 & $102512_{3 / 2}$ & $-175765_{5 / 2}^{\circ}$ & 1328.835 & 15 & 75253.88 & $\left\{102542_{7 / 2}\right.$ & $-177796_{7 / 2}^{\circ}$ \\
\hline 1365.005 & 600 & 73259.80 & $102875_{9 / 2}$ & $-176135_{11 / 2}^{\circ}$ & & & & $\left\{127045_{13 / 2}\right.$ & $-202299_{13 / 2}^{\circ}$ \\
\hline 1364.484 & 600 & 73287.77 & $98222_{5 / 2}$ & $-171510_{7 / 2}^{\circ}$ & 1326.362 & 8000 & 75394.19 & $95580_{15 / 2}$ & $-170974_{13 / 2}^{\circ}$ \\
\hline 1364.399 & 1500 & 73292.34 & & & 1326.319 & 2000 & 75396.64 & & \\
\hline
\end{tabular}


TABLE 1. Classified spectral lines of $\mathrm{Yb} \mathrm{IV.}$

Wavelengths longer than $2000 \AA$ are values in air-continued

\begin{tabular}{|c|c|c|c|c|c|c|c|c|c|}
\hline $\begin{array}{c}\text { Wavelength } \\
(\AA)\end{array}$ & Intensity & $\begin{array}{c}\text { Wavenumber } \\
\left(\mathrm{cm}^{-1}\right)\end{array}$ & Classific & ication & $\begin{array}{c}\text { Wavelength } \\
\text { (§) }\end{array}$ & Intensity & $\begin{array}{c}\text { Wavenumber } \\
\left(\mathrm{cm}^{-1}\right)\end{array}$ & Classif & ification \\
\hline 1324.608 & 20 & 75494.03 & $102542_{7 / 2}$ & $-178036_{7 / 2}^{\circ}$ & 1261.531 & 400 & 79268.76 & $88322_{5 / 2}$ & $-167590_{5 / 2}^{\circ}$ \\
\hline 1324.375 & 100 & 75507.31 & $102542_{7 / 2}$ & $-178049_{5 / 2}^{\circ}$ & 1261.218 & 100 & 79288.43 & & \\
\hline 1323.921 & 300 & 75533.20 & $95977_{7 / 2}$ & $-171510_{7 / 2}^{\circ}$ & 1260.332 & 60 & 79344.17 & $99180_{11 / 2}$ & $-178524_{\mathbf{g} / 2}^{\circ}$ \\
\hline 1323.855 & 1 & 75536.97 & $102512_{3 / 2}$ & $-178049_{5 / 2}^{\circ}$ & 1259.889 & 1 & 79372.07 & $90045_{9 / 2}$ & $-169417_{7 / 2}^{\circ}$ \\
\hline 1323.366 & 150 & 75564.88 & $85545_{13 / 2}$ & $-161110_{15 / 2}^{\circ}$ & 1259.625 & 20 & 79388.70 & $82673_{11 / 2}$ & $-162061_{11 / 2}^{\circ}$ \\
\hline 1321.886 & 10 & 75649.48 & $102875_{9 / 2}$ & $-178524_{9 / 2}^{\circ}$ & 1253.100 & 2 & 79802.09 & $88322_{5 / 2}$ & $-168124_{7 / 2}^{\circ}$ \\
\hline 1321.668 & 2 & 75661.96 & & & 1252.403 & 60 & 79846.50 & $88175_{11 / 2}$ & $-16802 l_{11 / 2}^{\circ}$ \\
\hline 1317.886 & 6 & 75879.09 & $102158_{5 / 2}$ & $-178036_{7 / 2}^{\circ}$ & 1251.511 & 50 & 79903.41 & $88118_{9 / 2}$ & $-168021_{11 / 2}^{\circ}$ \\
\hline 1317.857 & 20 & 75880.76 & $91532_{7 / 2}$ & $-167413_{7 / 2}^{\circ}$ & 1251.286 & 15 & 79917.78 & $88175_{11 / 2}$ & $-168092_{9 / 2}^{\circ}$ \\
\hline 1317.703 & 100 & 75889.63 & $95977_{7 / 2}$ & $-171867_{9 / 2}^{\circ}$ & 1250.595 & 1000 & 79961.93 & $82673_{11 / 2}$ & $-162635_{13 / 2}^{\circ}$ \\
\hline 1317.582 & 20 & 75896.60 & $95078_{11 / 2}$ & $-170974_{13 / 2}^{\circ / 2}$ & 1250.362 & 40 & 79976.83 & $91532_{7 / 2}$ & $-171510_{7 / 2}^{\circ}$ \\
\hline 1316.986 & 800 & 75930.95 & $91482_{5 / 2}$ & $-167413_{7 / 2}^{\circ}$ & 1249.660 & 100 & 80021.76 & $98502_{7 / 2}$ & $-178524_{\mathbf{g} / 2}^{9}$ \\
\hline 1316.040 & 9000 & 75985.53 & $85124_{17 / 2}$ & $-161110_{15 / 2}^{\circ}$ & 1249.561 & 80 & 80028.10 & $91482_{5 / 2}$ & $-171510_{7 / 2}^{\circ}$ \\
\hline 1314.794 & 150 & 76057.54 & $91532_{7 / 2}$ & $-167590_{5 / 2}^{\circ}$ & 1245.945 & 1 & 80260.36 & & \\
\hline 1314.240 & 1 & 76089.60 & $91931_{13 / 2}$ & $-16802 l_{11 / 2}^{0 / 2}$ & 1245.649 & 200 & 80279.43 & $91931_{13 / 2}$ & $-172211_{11 / 2}^{\circ}$ \\
\hline 1313.920 & 100 & 76108.13 & $91482_{5 / 2}$ & $-167590_{5 / 2}^{\circ}$ & 1244.803 & 30 & 80333.99 & $\left\{98971_{9 / 2}\right.$ & $-179305_{7 / 2}^{\circ}$ \\
\hline 1313.568 & 20 & 76128.52 & $100350_{7 / 2}$ & $-176478_{9 / 2}^{\circ}$ & & & & $\left\{91532_{7 / 2}\right.$ & $-171867_{9 / 2}^{\circ}$ \\
\hline 1312.469 & 150 & 76192.27 & $100350_{7 / 2}$ & $-176542_{7 / 2}^{8 / 2}$ & 1244.180 & 100 & 80374.22 & & \\
\hline 1311.883 & 1 & 76226.31 & & & 1243.619 & 60 & 80410.47 & $78529_{9 / 2}$ & $-158939_{9 / 2}^{\circ}$ \\
\hline 1311.195 & 100 & 76266.30 & $82673_{11 / 2}$ & $-158939_{9 / 2}^{\circ}$ & 1243.015 & 600 & 80449.55 & $82185_{15 / 2}$ & $-162635_{13 / 2}^{\circ}$ \\
\hline 1308.548 & 100 & 76420.58 & $99344_{5 / 2}$ & $-175765_{5 / 2}^{\circ / 2}$ & 1242.207 & 1 & 80501.88 & $95977_{7 / 2}$ & $-176478_{9 / 2}^{\circ}$ \\
\hline 1307.943 & 10 & 76455.93 & $109413_{5 / 2}$ & $-185869_{5 / 2}^{0 / 2}$ & 1237.976 & 3 & 80777.01 & $105685_{7 / 2}$ & $-186462_{9 / 2}^{\circ}$ \\
\hline 1307.712 & 800 & 76469.43 & & & 1237.830 & 6 & 80786.53 & & \\
\hline 1306.919 & 600 & 76515.83 & $85545_{13 / 2}$ & $-162061_{11 / 2}^{\circ}$ & 1237.571 & 1 & 80803.44 & $98502_{7 / 2}$ & $-179305_{7 / 2}^{\circ}$ \\
\hline 1306.740 & 1 & 76526.31 & $102158_{5 / 2}$ & $-178684_{5 / 2}^{0}$ & 1234.839 & 300 & 80982.21 & & \\
\hline 1306.192 & 800 & 76558.42 & $\left\{101966_{9 / 2}^{5 / 2}\right.$ & $-178524_{9 / 2}^{\circ}$ & 1233.115 & 2 & 81095.43 & $88322_{5 / 2}$ & $-169417_{7 / 2}^{\circ}$ \\
\hline & & & $\left\{109413_{5 / 2}\right.$ & $-185972_{7 / 2}^{\circ}$ & 1230.491 & 500 & 81268.37 & $10214_{5 / 2}^{\circ}$ & $-\quad 91482_{5 / 2}$ \\
\hline 1305.584 & 2000 & 76594.07 & $107873_{5 / 2}$ & $-184467_{3 / 2}^{\circ}$ & 1229.732 & 1000 & 81318.53 & $10214_{5 / 2}^{\circ}$ & $-\quad 91532_{7 / 2}$ \\
\hline 1304.733 & 250 & 76644.03 & & & 1228.328 & 1 & 81411.47 & $88322_{5 / 2}$ & $-169733_{3 / 2}^{\circ}$ \\
\hline 1303.965 & 2 & 76689.17 & $115984_{7 / 2}$ & $-192674_{5 / 2}^{\circ}$ & 1228.064 & 1 & 81428.98 & & \\
\hline 1302.815 & 60 & 76756.86 & & & 1226.207 & 1 & 81552.29 & $89422_{13 / 2}$ & $-170974_{13 / 2}^{\circ}$ \\
\hline 1302.712 & 20 & 76762.93 & $102542_{7 / 2}$ & $-179305_{7 / 2}^{\circ}$ & 1224.818 & 1 & 81644.78 & $94834_{7 / 2}$ & $-176478_{9 / 2}^{\circ}$ \\
\hline 1301.916 & 1 & 76809.87 & & & 1221.087 & 4 & 81894.24 & & \\
\hline 1300.692 & 1 & 76882.15 & $109025_{\mathbf{9} / \mathbf{2}}$ & $-185907_{11 / 2}^{\circ}$ & 1216.579 & 2 & 82197.70 & $102158_{5 / 2}$ & $-184355_{5 / 2}^{\circ}$ \\
\hline 1300.491 & 2 & 76894.03 & $95316_{13 / 2}$ & $-172211_{11 / 2}^{\circ}$ & 1212.479 & 200 & 82475.65 & $85545_{13 / 2}$ & $-168021_{11 / 2}^{\circ}$ \\
\hline 1299.591 & 20 & 76947.28 & $109025_{9 / 2}^{13 / 2}$ & $-185972_{7 / 2}^{\circ}$ & 1211.421 & 3 & 82547.68 & $95977_{7 / 2}$ & $-178524_{9 / 2}^{\circ}$ \\
\hline 1298.500 & 20 & 77011.93 & $94498_{5 / 2}$ & $-171510_{7 / 2}^{\circ}$ & 1210.452 & 3 & 82613.76 & & \\
\hline 1298.236 & 50 & 77027.59 & & & 1207.513 & 20 & 82814.84 & & \\
\hline 1298.155 & 100 & 77032.40 & $94834_{7 / 2}$ & $-171867_{9 / 2}^{\circ}$ & 1207.016 & 20 & 82848.94 & $84347_{7 / 2}$ & $-167196_{9 / 2}^{\circ}$ \\
\hline 1297.192 & 300 & 77089.59 & $85545_{13 / 2}$ & $-162635_{13 / 2}^{\circ / 2}$ & 1201.695 & 20 & 83215.79 & $94834_{7 / 2}$ & $-178049_{5 / 2}^{\circ}$ \\
\hline 1296.461 & 40 & 77133.05 & $95078_{11 / 2}$ & $-172211_{11 / 2}^{\circ}$ & 1200.336 & 1 & 83310.00 & & \\
\hline 1296.166 & 20 & 77150.61 & $90045_{9 / 2}^{11 / 2}$ & $-167196_{9 / 2}^{\circ}$ & 1197.133 & 250 & 83532.90 & $78529_{9 / 2}$ & $-162061_{11 / 2}^{\circ}$ \\
\hline 1295.936 & 500 & 77164.30 & & & 1193.637 & 2 & 83777.56 & $84347_{7 / 2}$ & $-168124_{7 / 2}^{\circ}$ \\
\hline 1295.551 & 400 & 77187.23 & $90834_{11 / 2}$ & $-168021_{11 / 2}^{\circ}$ & 1189.156 & 1 & 84093.25 & $88118_{9 / 2}$ & $-172211_{11 / 2}^{\circ}$ \\
\hline 1294.355 & 20 & 77258.55 & $90834_{11 / 2}$ & $-168092_{9 / 2}^{\circ}$ & 1186.454 & 5 & 84284.76 & $10214_{5 / 2}^{\circ / 2}$ & $\begin{array}{l}-\quad 94498_{5 / 2} \\
-\quad 1 / 2\end{array}$ \\
\hline 1294.290 & 150 & 77262.43 & $98502_{7 / 2}$ & $-175765_{5 / 2}^{\circ}$ & 1185.579 & 6000 & 84346.97 & $0_{7 / 2}^{\circ}$ & $-84347_{7 / 2}$ \\
\hline 1292.725 & 400 & 77355.97 & & & 1183.807 & 10 & 84473.22 & $78529_{9 / 2}$ & $-163002_{11 / 2}^{\circ}$ \\
\hline 1291.957 & 25 & 77401.95 & $124897_{11 / 2}$ & $-202299_{13 / 2}^{\circ}$ & 1181.751 & 1000 & 84620.19 & $10214_{5 / 2}^{\circ}$ & $-\quad 94834_{7 / 2}$ \\
\hline 1291.762 & 400 & 77413.64 & & & 1166.012 & 500 & 85762.41 & $10214_{5 / 2}^{\circ / 2}$ & $95977_{7 / 2}$ \\
\hline 1291.572 & 25 & 77425.02 & $115984_{7 / 2}$ & $-193409_{7 / 2}^{\circ}$ & 1162.278 & 5 & 86037.93 & & \\
\hline 1291.227 & 200 & 77445.71 & $100350_{7 / 2}$ & $-177796_{7 / 2}^{\circ / 2}$ & 1155.330 & 3 & 86555.35 & & \\
\hline 1290.245 & 2000 & 77504.66 & $122222_{17 / 2}$ & $-199726_{15 / 2}^{\circ}$ & 1136.237 & 300 & 88009.80 & $10214_{5 / 2}^{\circ}$ & $98222_{5 / 2}$ \\
\hline 1289.619 & 1 & 77542.28 & $98222_{5 / 2}$ & $-175765_{5 / 2}^{\circ}$ & 1134.426 & 5000 & 88150.30 & & \\
\hline 1289.130 & 400 & 77571.69 & $9897 l_{9 / 2}$ & $-176542_{7 / 2}^{\circ / 2}$ & 1128.894 & 20 & 88582.27 & & \\
\hline 1287.402 & 4 & 77675.81 & $89520_{7 / 2}$ & $-167196_{9 / 2}^{\circ}$ & 1122.529 & 60 & 89084.55 & & \\
\hline 1287.009 & 1 & 77699.53 & $100350_{7 / 2}$ & $-178049_{5 / 2}^{\circ / 2}$ & 1121.943 & 20 & 89131.08 & $10214_{5 / 2}^{\circ}$ & $99344_{5 / 2}$ \\
\hline 1285.378 & 15 & 77798.12 & & & 1117.064 & 100 & 89520.38 & $0_{7 / 2}^{\circ}$ & $89520_{7 / 2}$ \\
\hline 1281.388 & 6 & 78040.37 & $98502_{7 / 2}$ & $-176542_{7 / 2}^{\circ}$ & 1110.548 & 200 & 90045.63 & $0_{7 / 2}^{\circ}$ & $-\quad 90045_{9 / 2}$ \\
\hline 1280.897 & 2 & 78070.28 & $89520_{7 / 2}$ & $-167590_{5 / 2}^{\circ}$ & 1109.431 & 100 & 90136.29 & $10214_{5 / 2}^{\circ}$ & $-100350_{7 / 2}$ \\
\hline 1280.758 & 1 & 78078.76 & $90045_{9 / 2}$ & $-168124_{7 / 2}^{\mathrm{o} / 2}$ & 1107.680 & 5 & 90278.78 & & \\
\hline 1280.284 & 1500 & 78107.67 & $10214_{5 / 2}^{8 / 2}$ & $-\quad 88322_{5 / 2}$ & 1097.643 & 100 & 91104.30 & $10214_{5 / 2}^{\circ}$ & $-101318_{5 / 2}$ \\
\hline 1276.580 & 2 & 78334.29 & $100350_{7 / 2}$ & $-178684_{5 / 2}^{0 / 2}$ & 1093.109 & 50 & 91482.18 & $0_{7 / 2}^{\circ}$ & $-\quad 91482_{5 / 2}$ \\
\hline 1276.202 & 4 & 78357.50 & & & 1092.510 & 400 & 91532.34 & $0_{7 / 2}^{\circ}$ & $-\quad 91532_{7 / 2}$ \\
\hline 1273.121 & 6 & 78547.12 & $97687_{11 / 2}$ & $-176135_{11 / 2}^{\circ}$ & 1087.621 & 50 & 91943.79 & $10214_{5 / 2}^{\circ}$ & $-102158_{5 / 2}$ \\
\hline 1272.794 & 2 & 78567.30 & & & 1083.441 & 50 & 92298.52 & $10214_{5 / 2}^{0}$ & $-102512_{3 / 2}$ \\
\hline 1272.284 & 400 & 78598.80 & $89422_{13 / 2}$ & $-168021_{11 / 2}^{\circ}$ & 1083.088 & 2 & 92328.60 & $10214_{5 / 2}^{\mathrm{C}}$ & $-102542_{7 / 2}$ \\
\hline 1269.209 & 3 & 78789.22 & & & 1072.155 & 20 & 93270.09 & $10214_{5 / 2}^{\circ / 2}$ & $-103484_{5 / 2}$ \\
\hline 1269.048 & 10 & 78799.22 & & & 1063.694 & 40 & 94011.99 & $10214_{5 / 2}^{\mathrm{o} / 2}$ & $-104225_{7 / 2}$ \\
\hline 1267.570 & 80 & 78891.10 & $97587_{11 / 2}$ & $-176478_{9 / 2}^{\circ}$ & 1059.468 & 100 & 94386.99 & $0_{7 / 2}^{\circ}$ & $-\quad 94386_{9 / 2}$ \\
\hline 1267.028 & 300 & 78924.85 & $82185_{15 / 2}$ & $-161110_{15 / 2}^{\circ}$ & 1058.223 & 50 & 94498.03 & $0_{7 / 2}^{\circ}$ & $94498_{5 / 2}$ \\
\hline 1265.993 & 1 & 78989.37 & & & 1054.463 & 1000 & 94835.00 & $0_{7 / 2}^{\circ}$ & $94834_{7 / 2}$ \\
\hline 1265.134 & 20 & 79043.01 & $91931_{13 / 2}$ & $-170974_{13 / 2}^{\circ}$ & 1050.245 & 1000 & 95215.87 & $0_{7 / 2}^{\circ}$ & $-\quad 95215_{9 / 2}$ \\
\hline 1263.593 & 15 & 79139.40 & & & 1047.433 & 3 & 95471.50 & $10214_{5 / 2}^{\circ / 2}$ & $-105685_{7 / 2}$ \\
\hline 1263.141 & 80 & 79167.72 & & & 1043.558 & 40 & 95826.01 & $10214_{5 / 2}^{\circ}$ & $-106039_{7 / 2}$ \\
\hline
\end{tabular}


TABLE 1. Classified spectral lines of $\mathrm{Yb}$ IV.

Wavelengths longer than $2000 \mathrm{~A}$ are values in air-continued

\begin{tabular}{|c|c|c|c|c|c|c|c|c|c|}
\hline \multirow{2}{*}{$\begin{array}{c}\begin{array}{c}\text { Wavelength } \\
(\AA)\end{array} \\
1041.917\end{array}$} & \multirow{2}{*}{$\begin{array}{c}\text { Intensity } \\
1\end{array}$} & $\begin{array}{c}\text { Wavenumber } \\
\left(\mathrm{cm}^{-1}\right)\end{array}$ & \multicolumn{2}{|c|}{ Classification } & \multirow{2}{*}{$\begin{array}{c}\begin{array}{c}\text { Wavelength } \\
(\AA)\end{array} \\
908.141\end{array}$} & \multirow{2}{*}{\begin{tabular}{c|} 
Intensity \\
10
\end{tabular}} & \multirow{4}{*}{\begin{tabular}{c|}
$\begin{array}{c}\text { Wavenumber } \\
\left(\mathrm{cm}^{-1}\right)\end{array}$ \\
110115.05 \\
110468.67 \\
110558.93
\end{tabular}} & \multicolumn{2}{|c|}{ Classification } \\
\hline & & 95976.93 & $0_{7 / 2}^{\circ}$ & $-95977_{7 / 2}$ & & & & $10214_{5 / 2}^{\circ}$ & $-120328_{5 / 2}$ \\
\hline 1040.732 & 50 & 96086.21 & $95580_{15 / 2}$ & $-191666_{13 / 2}^{\circ}$ & 905.234 & 1 & & & \\
\hline 1033.426 & 3 & 96765.51 & & & 904.495 & 10 & & & \\
\hline 1024.323 & 100 & 97625.45 & & & 902.456 & 300 & 110808.72 & $0_{7 / 2}^{\circ}$ & $-110808_{7 / 2}$ \\
\hline 1018.089 & 30 & 98223.23 & $0_{7 / 2}^{\circ}$ & $-98222_{5 / 2}$ & 896.455 & 40 & 111550.49 & $0_{7 / 2}^{\circ}$ & $-111550_{7 / 2}$ \\
\hline 1015.206 & 10 & 98502.17 & $0_{7 / 2}^{\circ}$ & $-\quad 98502_{7 / 2}$ & 891.095 & 2 & 112221.47 & & \\
\hline 1010.391 & 50 & 98971.58 & $0_{7 / 2}^{\circ}$ & $-\quad 98971_{9 / 2}$ & 887.474 & 5 & 112679.35 & $0_{7 / 2}^{\circ}$ & $-112679_{5 / 2}$ \\
\hline 1008.066 & 40 & 99199.85 & $10214_{5 / 2}^{0}$ & $-109413_{5 / 2}$ & 886.429 & 20 & 112812.19 & $0_{7 / 2}^{\circ / 2}$ & $-112812_{9 / 2}^{5 / 2}$ \\
\hline 1006.592 & 5 & 99345.11 & $0_{7 / 2}^{\circ}$ & $-\quad 99344_{5 / 2}$ & 875.159 & 20 & 114264.95 & & \\
\hline 996.503 & 20 & 100350.92 & $0_{7 / 2}^{\circ}$ & $-100350_{7 / 2}$ & 871.646 & 20 & 114725.47 & $10214_{5 / 2}^{\circ}$ & $-124939_{5 / 2}$ \\
\hline 994.081 & 100 & 100595.42 & $10214_{5 / 2}^{0}$ & $-110808_{7 / 2}$ & 871.076 & 1 & 114800.54 & & \\
\hline 986.983 & 50 & 101318.86 & $0_{7 / 2}^{\circ}$ & $-101318_{5 / 2}$ & 870.352 & 200 & 114896.04 & $10214_{5 / 2}^{\circ}$ & $-125110_{7 / 2}$ \\
\hline 986.808 & 10 & 101336.83 & $10214_{5 / 2}^{\circ}$ & $-111550_{7 / 2}$ & 860.694 & 2 & 116185.31 & $10214_{5 / 2}^{\mathrm{O}}$ & $-126399_{5 / 2}$ \\
\hline 980.708 & 50 & 101967.14 & $0_{7 / 2}^{\circ}$ & $-101966_{9 / 2}$ & 857.243 & 10 & 116653.03 & $10214_{5 / 2}^{\mathrm{o} / 2}$ & $-126867_{7 / 2}^{3 / 2}$ \\
\hline 978.879 & 30 & 102157.67 & $0_{7 / 2}^{\circ}$ & $-102157_{5 / 2}$ & 854.513 & 40 & 117025.72 & $0_{7 / 2}^{\circ}$ & $-117026_{5 / 2}$ \\
\hline 975.979 & 100 & 102461.22 & $10214_{5 / 2}^{\circ}$ & $-112675_{3 / 2}$ & 842.548 & 10 & 118687.60 & $10214_{5 / 2}^{\circ}$ & $-128901_{7 / 2}$ \\
\hline 975.944 & 100 & 102464.89 & $10214_{5 / 2}^{\circ}$ & $-112679_{5 / 2}$ & 842.336 & 5 & 118717.47 & $0_{7 / 2}^{\circ}$ & $-118717_{7 / 2}$ \\
\hline 975.206 & 200 & 102542.43 & $0_{7 / 2}^{\circ}$ & $-102542_{7 / 2}$ & 841.480 & 1 & 118838.23 & & \\
\hline 972.859 & 40 & 102789.81 & $88877_{15 / 2}$ & $-191666_{13 / 2}^{\circ}$ & 835.305 & 2 & 119716.75 & & \\
\hline 966.332 & 4 & 103484.10 & $0_{7 / 2}$ & $-103484_{5 / 2}^{10 / 2}$ & 831.056 & 40 & 120328.83 & $0_{7 / 2}^{\circ}$ & $-120328_{5 / 2}$ \\
\hline 959.454 & 100 & 104225.94 & $0_{7 / 2}$ & $-104255_{7 / 2}^{5 / 2}$ & 828.957 & 200 & 120633.51 & $0_{7 / 2}^{\circ}$ & $-120633_{9 / 2}$ \\
\hline 955.907 & 10 & 104612.68 & $0_{7 / 2}$ & $-104613_{9 / 2}$ & 827.994 & 20 & 120773.82 & $0_{7 / 2}^{\circ}$ & $-120773_{9 / 2}$ \\
\hline 946.781 & 20 & 105621.04 & & & 826.391 & 40 & 121008.09 & $0_{7 / 2}^{\circ}$ & $-121008_{9 / 2}^{9 / 2}$ \\
\hline 946.205 & 400 & 105685.34 & $0_{7 / 2}^{\circ}$ & $-105685_{7 / 2}$ & 823.426 & 30 & 121443.82 & & \\
\hline 945.440 & 10 & 105770.85 & $10214_{5 / 2}^{0 / 2}$ & $-115984_{7 / 2}^{7 / 2}$ & 819.641 & 3 & 122004.63 & & \\
\hline 943.039 & 400 & 106040.15 & $0_{7 / 2}^{\circ}$ & $-106039_{7 / 2}$ & 818.816 & 20 & 122127.55 & & \\
\hline 936.221 & 300 & 106812.38 & $10214_{5 / 2}^{0}$ & $-117026_{5 / 2}$ & 816.515 & 100 & 122471.72 & & \\
\hline 932.529 & 1 & 107235.27 & & & 816.485 & 2 & 122476.22 & $0_{7 / 2}^{\circ}$ & $-122476_{5 / 2}$ \\
\hline 929.660 & 50 & 107566.20 & $0_{7 / 2}^{\circ}$ & $-107566_{9 / 2}$ & 800.386 & 40 & 124939.71 & $0_{7 / 2}^{\circ}$ & $-124939_{5 / 2}$ \\
\hline 927.719 & 2 & 107791.26 & & & 791.143 & 10 & 126399.39 & $0_{7 / 2}^{\circ}$ & $-126399_{5 / 2}$ \\
\hline 927.013 & 300 & 107873.35 & $0_{7 / 2}^{\circ}$ & $-107873_{5 / 2}$ & 788.225 & 25 & 126867.32 & $0_{7 / 2}^{\circ}$ & $-126867_{7 / 2}^{5 / 2}$ \\
\hline 918.740 & 1 & 108844.72 & & & 775.787 & 300 & 128901.35 & $0_{7 / 2}^{\circ}$ & $-128901_{7 / 2}$ \\
\hline 917.221 & 2 & 109024.97 & $0_{7 / 2}^{\circ}$ & $-109025_{9 / 2}$ & 761.059 & 15 & 131395.86 & $0_{7 / 2}^{\circ}$ & $-131395_{7 / 2}$ \\
\hline 913.338 & 1 & 109488.49 & & & & & & & \\
\hline
\end{tabular}


TABLE II. Observed energy levels of triply ionized ytterbium in units of $\mathrm{cm}^{-1}$. Term assignments are generally obtained from the major component of the eigenvector.

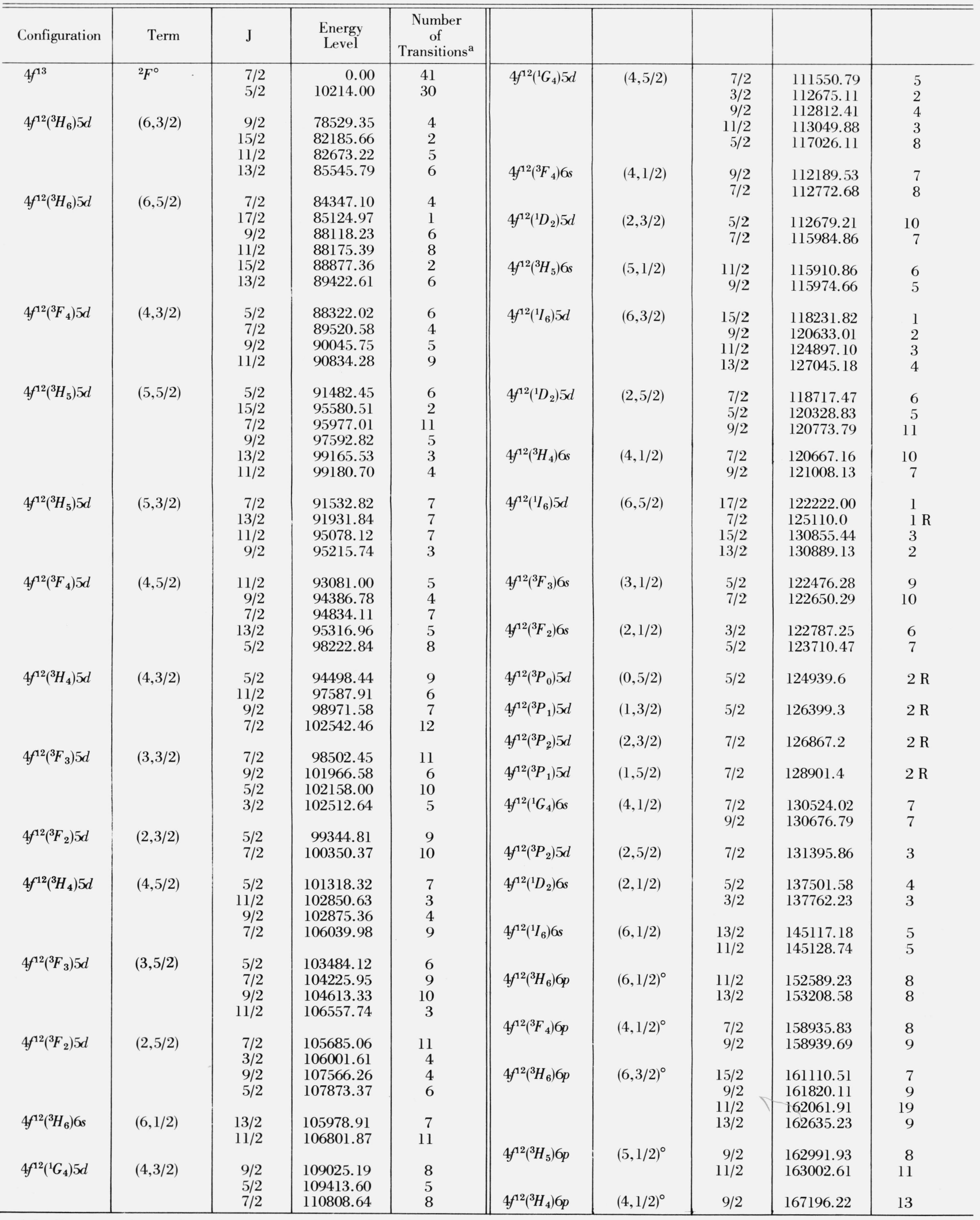

a An $\mathrm{R}$ next to the number of transitions indicates that this level is derived solely from resonance transition(s). 
TABLE II. Observed energy levels of triply ionized ytterbium in units of $\mathrm{cm}^{-1}$.

Term assignments are generally obtained from the major component of the eigenvector-continued

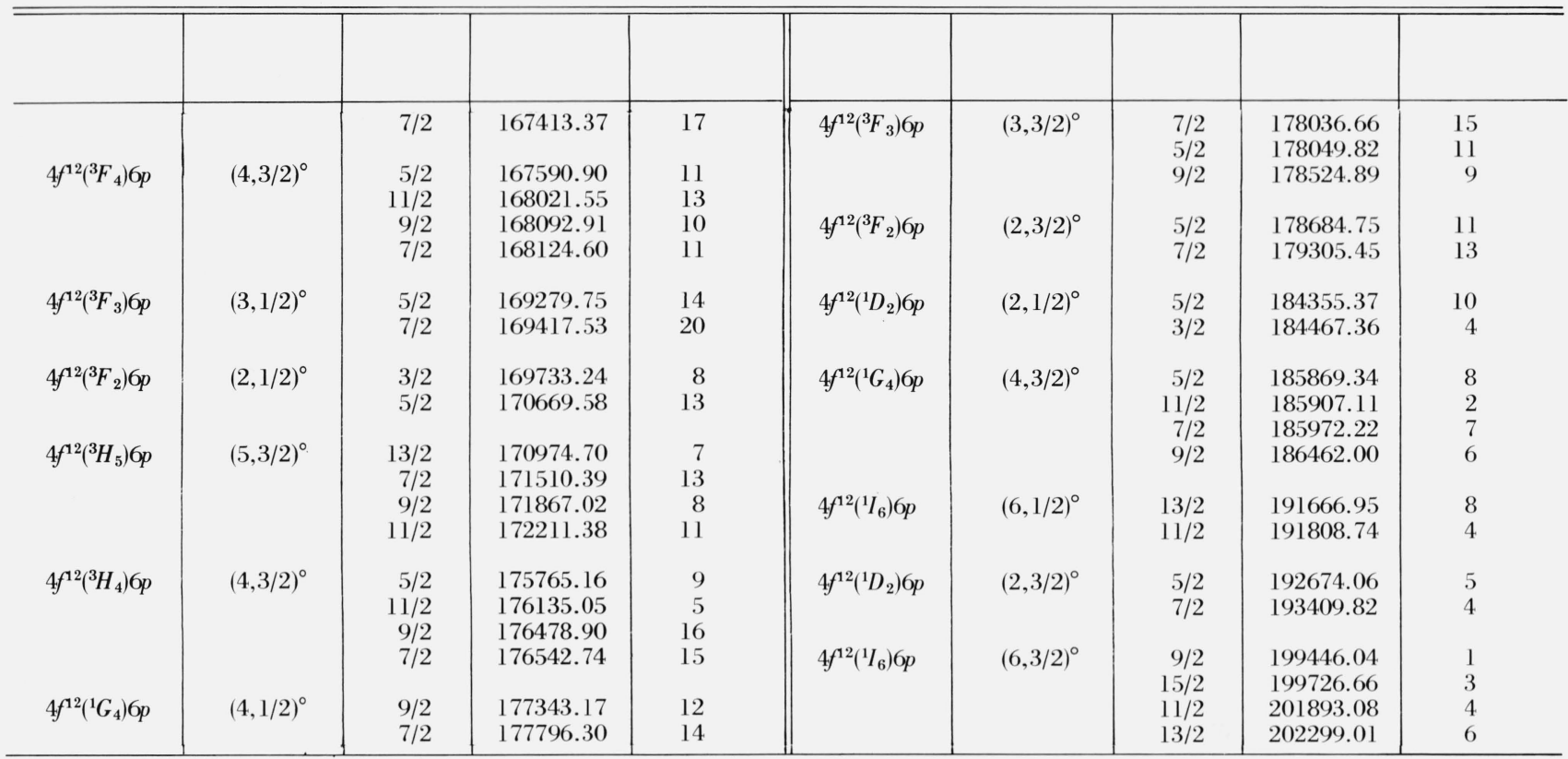

TABLE III. Fitted values for radial parameters of the $4 \mathrm{f}^{12} 5 \mathrm{~d}, 4 \mathrm{f}^{12} 6 \mathrm{~s}$, and $4 \mathrm{f}^{12} 6 \mathrm{p}$ configurations of $\mathrm{Yb}$ IV. Units are $\mathrm{cm}^{-1}$.

\begin{tabular}{|c|c|c|c|}
\hline Parameter & $4 f^{12} 5 d$ & $4 f^{12} 6 s$ & $4 f^{12} 6 p$ \\
\hline $\begin{array}{l}\text { A } \\
E^{1}\left(f^{12}\right) \\
E^{2}\left(f^{12}\right) \\
E^{3}\left(f^{12}\right) \\
\alpha\left(f^{12}\right) \\
\beta\left(f^{12}\right) \\
\gamma\left(f^{12}\right) \\
\zeta(4 f) \\
F^{2}(f d) \\
F^{4}(f d) \\
G^{1}(f d) \\
G^{3}(f d) \\
G^{5}(f d) \\
D^{1}(f d) \\
D^{3}(f d) \\
X^{2}(f d) \\
X^{4}(f d) \\
\zeta(5 d) \\
F^{2}(f p) \\
G^{2}(f p) \\
G^{4}(f p) \\
D^{1}(f p) \\
X^{3}(f p) \\
\zeta(6 p) \\
G^{3}(f s) \\
R^{2}(f d, f s)^{\mathrm{a}} \\
R^{3}(f d, s f)^{\mathrm{a}}\end{array}$ & $\begin{array}{r}108872 \pm 110 \\
7684 \pm 21 \\
36.83 \pm .18 \\
758.9 \pm 1.9 \\
21.6 \pm 1.4 \\
-1000 \text { (fixed) } \\
-52.7 \pm 6.2 \\
3079.6 \pm 5.3 \\
24434 \pm 110 \\
16545 \pm 280 \\
8454 \pm 34 \\
9760 \pm 260 \\
6465 \pm 240 \\
-868 \pm 100 \\
0 \text { (fixed) } \\
-2677 \pm 220 \\
-2770 \pm 340 \\
1752.3 \pm 8.5\end{array}$ & $\begin{array}{r}121758 \pm 40 \\
7771 \pm 66 \\
37.73 \pm .37 \\
752.7 \pm 7.9 \\
18.5 \pm 2.5 \\
-1000 \text { (fixed) } \\
-57 \text { (fixed) } \\
3065.1 \pm 8.7\end{array}$ & $\begin{array}{r}176104 \pm 38 \\
7806 \pm 17 \\
37.99 \pm .09 \\
752.0 \pm 1.9 \\
17.3 \pm .7 \\
-1000 \text { (fixed) } \\
-59.0 \pm 2.3 \\
3065.7 \pm 2.1\end{array}$ \\
\hline
\end{tabular}


TABLE IV. Calculated energies and compositions of the levels of the $4 \mathrm{f}^{12}(5 \mathrm{~d}+6 \mathrm{~s})$ configuration of triply ionized ytterbium. Units are $\mathrm{cm}^{-1}$.

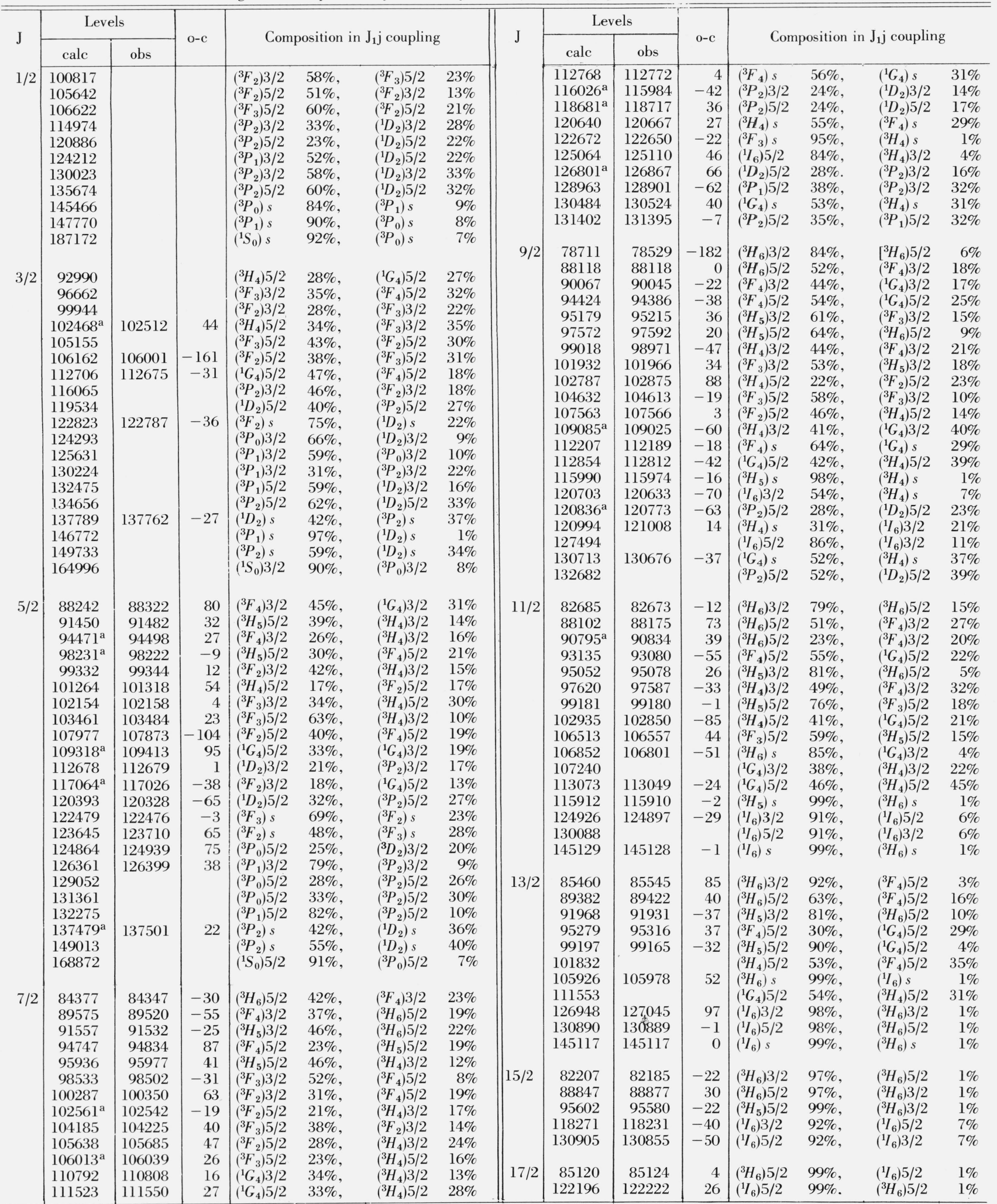

a The smaller of the listed eigenvectors was used in Table II as the level name. This was done in order to give each level a unique designation. 
TABLE V. Calculated energies and compositions of the levels of the $4 \mathrm{f}^{12} 6 \mathrm{p}$ configuration of the triply ionized ytterbium. Units are $\mathrm{cm}^{-1}$.

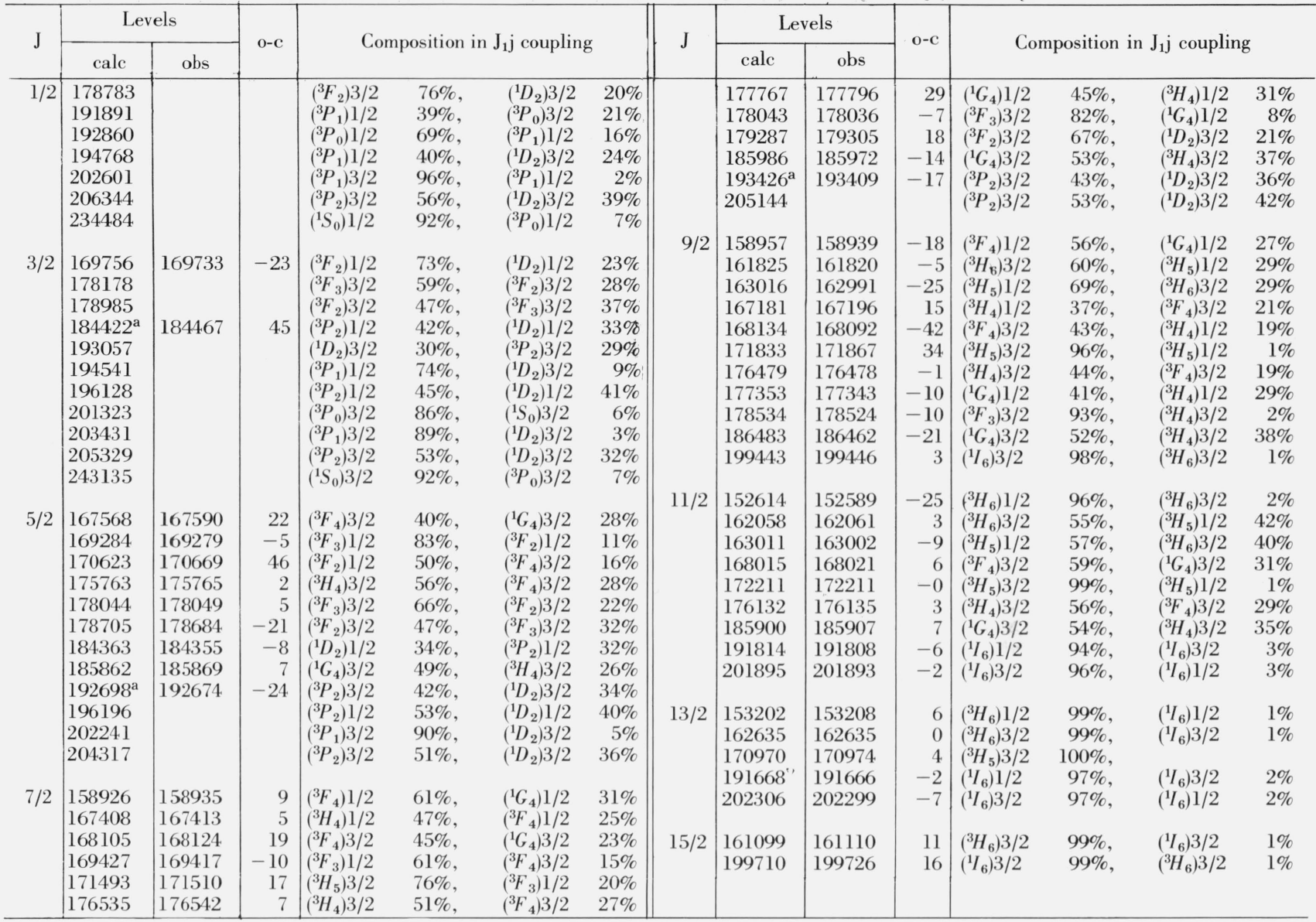

a The smaller of the listed eigenvectors was used in Table II as the level name. This was done in order to give each level a unique designation. 